\title{
Open clusters
}

\section{Fundamental parameters of B stars in NGC 3766 and NGC $4755^{\star, \star \star}$}

\author{
Y. Aidelman ${ }^{1}$, L. S. Cidale ${ }^{1, \star \star \star}$, J. Zorec ${ }^{2}$, and M. L. Arias ${ }^{1, \star \star \star}$ \\ 1 Departamento de Espectroscopía, Facultad de Ciencias Astronómicas y Geofísicas, Universidad Nacional de La Plata (UNLP) and \\ Instituto de Astrofísica La Plata, CCT La Plata, CONICET-UNLP, Paseo del Bosque S/N, 1900 La Plata, Argentina \\ e-mail: aidelman@fcaglp.unlp.edu.ar \\ ${ }^{2}$ Université Pierre \& Marie Curie, CNRS UMR 7095, Institut d'Astrophysique de Paris, $98^{\text {bis }}$ Bd. Arago, 75014 Paris, France
}

Received 17 February 2012 / Accepted 1 June 2012

\section{ABSTRACT}

\begin{abstract}
Context. Spectroscopic investigations of galactic open clusters are scarce and limited to a reduced sample of cluster members. Aims. We intend to perform a complete study of the physical parameters of two galactic clusters as well as of their individual members. Methods. To carry out this study, we used the BCD (Barbier-Chalonge-Divan) spectrophotometric system, which is based on the study of the Balmer discontinuity and is independent of interstellar and circumstellar extinction. Additional physical properties were derived from the line profiles (FWHM) and stellar evolution models. We analyzed low-resolution spectra around the Balmer discontinuity for normal B-type and Be stars in two open clusters: NGC 3766 and NGC 4755. We determined the stellar fundamental parameters, such as effective temperatures, surface gravities, spectral types, luminosity classes, absolute and bolometric magnitudes, and color gradient excesses. The stellar rotation velocity was also determined. Complementary information, mainly stellar mass, age, and radius of the star population were calculated using stellar evolution models. In some cases, the stellar fundamental parameters were derived for the first time. The obtained results allowed us also to determine the reddening, age, and distance to the clusters.

Results. The cluster parameters obtained through the BCD method agree very well with those derived from classical methods based on photometric data. The BCD system also provides physical properties of the star members. This study enables us to test the good behavior of $M_{\text {bol }}\left(\lambda_{1}, D\right)$-calibrations and detect systematic discrepancies between log $g$ estimates from model atmospheres and those derived from stellar evolution models. To improve our knowledge on the formation and evolution of the clusters, more statistical studies on the initial mass luminosity and angular momentum distributions should be addressed. Therefore, the BCD spectrophotometric system could be a powerful tool for studying far galactic and extragalactic clusters with the generation of large telescopes and the multi-object technique.
\end{abstract}

Key words. open clusters and associations: individual: NGC 3766 - stars: emission-line, Be - stars: fundamental parameters open clusters and associations: individual: NGC 4755 - stars: early-type

\section{Introduction}

The study of young open clusters is particularly interesting because they provide information about the structure and evolution of the galactic disk as well as evidence on the effects of the stellar evolution in the upper part of the Hertzsprung-Russell (HR) diagram. Nevertheless, among the 1787 currently known open clusters more than half have been poorly studied or remain even unstudied up to now (Piatti et al. 2011). From a statistical study of the published fundamental physical parameters for 395 open clusters, Paunzen \& Netopil (2006) reported that while the reddening measurements seemed to be quite accurate, the ages and distances suffered from severe uncertainties. For about $90 \%$ of all studied clusters, the error of the reddening was below 0.1 mag while absolute errors in the distance estimates were less than $20 \%$ for about $80 \%$ of the systems. However,

* Based on observations taken at CASLEO, operating under agreement of CONICET and the Universities of La Plata, Córdoba and San Juan, Argentina.

$\star \star$ Tables 7, 8, 12-14, 16-18 are available in electronic form at http: //www. aanda. org

$\star \star \star$ Member of the Carrera del Investigador Científico, CONICET, Argentina. only $11 \%$ of the investigated open clusters had errors of the ages of less than $20 \%$, although there were extreme discrepancies of more than $200 \%$.

On the other hand, spectroscopic investigations of galactic open clusters are quite scarce and are limited to a sample of cluster members that are primarily the Be stars and supergiant populations (Harris 1976; Mermilliod 1982; Slettebak 1985; Levesque et al. 2005). Our ignorance of the physical properties of the stellar population (temperature, gravity, luminosity) and the incidence of highly rotating stars and/or binary stars in open clusters leads to high difficulties in determinating the distance modulus, average reddening, and the ages of these systems. Thus, the estimated parameters of young galactic clusters are not only affected by the evolutionary stage of the massive stars, but also by rotation and other effects related to the $\mathrm{Be}$ and pulsation phenomena that modify the position of the stars in the HR diagram (Zorec et al. 2005). For instance, the dereddened colors of the Be stars are often subtracted from the observed average colors determined for the cluster, because a uniform reddening through the cluster area is assumed, and as a consequence only the interstellar effects can be removed. Therefore, there is a large amplitude scatter in the $U-B$ and $B-V$ color diagram caused by the Be peculiar colors, which makes the stars appear 


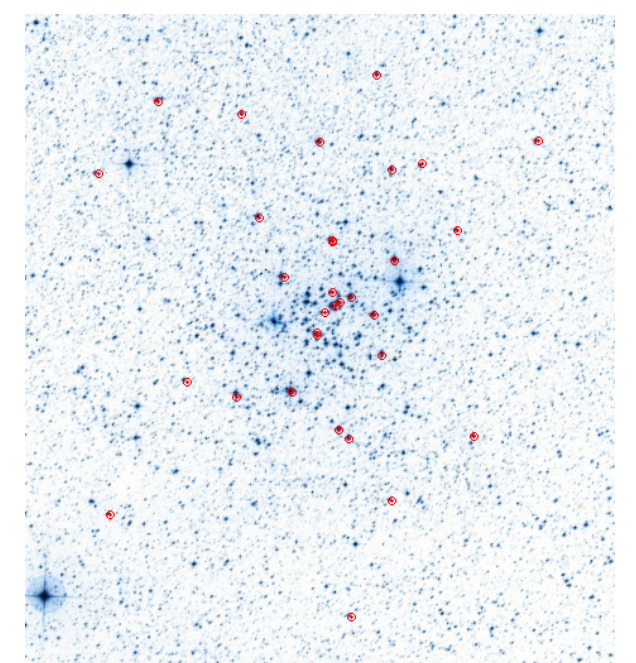

Fig. 1. Stars observed in NGC 3766.

more evolved. Our goal here is to carry out a complete study of the physical parameters of galactic clusters and their individual members using the BCD (Barbier-Chalonge-Divan) spectrophotometric system (Chalonge \& Divan 1973, 1977), which is based on the study of the Balmer discontinuity and it is independent of interstellar and circumstellar extinction. In this work we analyze low-resolution spectra around the Balmer discontinuity for normal B-type and Be stars in two open clusters: NGC 3766 and NGC 4755. We determine effective temperatures, surface gravities, spectral types, luminosity classes, absolute and bolometric magnitudes, and color gradient excess. Thus, we introduce an alternative method for determining the reddening, distance, and age to the clusters, since those parameters are traditionally obtained with photoelectric and photographic measurements in the $U B V$, ubvy and Geneva systems. This way, we show that the BCD system is an easy-to-use method for studying physical properties of galactic and extragalactic clusters.

The work is organized as follows: in Sect. 2 we describe the observations, Sect. 3 briefly explains the BCD method for deriving fundamental parameters of stars and clusters, and Sect. 4 shows the results obtained with the BCD method and from the stellar evolution theory. The discussion of the main results and our conclusions are given in Sects. 5 and 6, respectively.

\section{Observations}

We obtained low-resolution spectra of the brightest members of NGC 3766 and NGC 4755 (see Figs. 1 and 2) during multiple observing runs in 2002 January and 2003 June at the Complejo Astronómico El Leoncito (CASLEO), San Juan, Argentina, using the J. Sahade $2.15 \mathrm{~m}$ telescope with the Boller and Chivens spectrograph. The instrumental configuration was a $6001 \mathrm{~mm}^{-1}$ grating (\# 80), a slit width of $250 \mu \mathrm{m}$ and a CCD detector of $512 \times 512$ pixels. The obtained spectra cover the wavelength range 3500-4700 $\AA$ with an effective resolution of $4.53 \AA$ (which corresponds to two pixels) or $R=900$.

A standard reduction procedure was carried out to the spectroscopic images by applying overscan, bias- and flat-field corrections. Dark-frame subtractions were not necessary because we were dealing with bright stars with short exposure times. $\mathrm{He}-\mathrm{Ne}$-Ar comparison and spectrophotometric flux standard star spectra were also obtained to perform wavelength and flux calibrations. About two or four spectra of standard flux stars

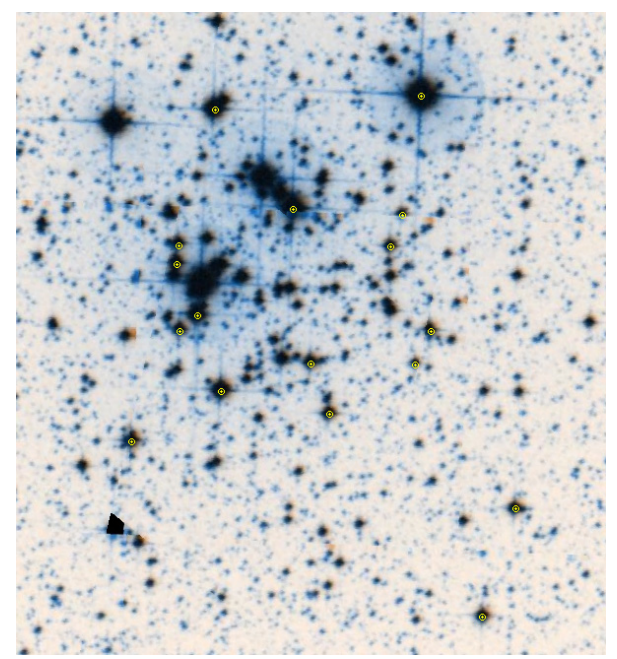

Fig. 2. Stars observed in NGC 4755.

(HR 3454, HR 7596, HR 5501 and/or HR 4468) were observed each night. Low-resolution spectra for the BCD spectrophotometry were obtained with the widest possible opening of the slit, to minimize light losses. We also note that observations were made at the lowest possible zenithal distance, to minimize refraction effects due to our atmosphere and thus to avoid light losses as a function of wavelength. The observations were reduced with the $\operatorname{IRAF}^{1}$ software package (version 2.14.1) and all spectra were corrected for atmospheric extinction.

The details of the observing runs of the 32 stars in NGC 3766 and 17 stars in NGC 4755 are summarized in Tables 1 and 2, respectively. The tables display the star identification, star coordinates, dates of observation, visual apparent magnitudes, and MK spectral type determinations with their respective references.

\section{The BCD spectrophotometric system}

\subsection{Fundamental parameters of the stars}

The fundamental parameters of the program stars $\left(T_{\mathrm{eff}}, \log g\right.$, $M_{\mathrm{v}}, M_{\mathrm{bol}}$, and spectral type) were derived using the BCD spectrophotometric system (Chalonge \& Divan 1973, 1977). This system is based on two observable parameters $\left(\lambda_{1}, D\right)$ that describe the Balmer discontinuity (BD): $D$ measures the height of the Balmer jump at $\lambda=3700 \AA$ in dex and is a strong indicator of the effective temperature; $\lambda_{1}$ gives the mean spectral position of the Balmer jump and is related to the surface gravity. A discussion and a detailed description of this method was presented in Zorec et al. (2009, Appendix A).

The BCD spectrophotometric system has several advantages, among which we highlight the following: 1) it is not affected either by interstellar or circumstellar extinction (Zorec \& Briot 1991), 2) it is based on measurable parameters of the visual continuum energy distribution near the BD that are thus related, on average, to physical properties of photospheric layers deeper than any classification system based on spectral line measurements, 3 ) it is possible to derive information on most of the fundamental parameters, 4) this method is easy and direct to apply,

\footnotetext{
1 IRAF is distributed by the National Optical Astronomy Observatory, which is operated by the Association of Universities for Research in Astronomy (AURA), Inc., under cooperative agreement with the National Science Foundation.
} 
Table 1. Stars in the open cluster NGC 3766. Nomenclature according to Ahmed (1962).

\begin{tabular}{|c|c|c|c|c|c|c|c|}
\hline $\begin{array}{l}\text { ID } \\
\text { Ahmed }\end{array}$ & $\begin{array}{c}\text { Other } \\
\text { designation }\end{array}$ & $\begin{array}{c}\alpha \\
(\mathrm{J} 2000)\end{array}$ & $\begin{array}{c}\delta \\
(\mathrm{J} 2000)\end{array}$ & $\begin{array}{c}\text { Observation } \\
\text { date }\end{array}$ & $m_{\mathrm{v}}$ & S.T & Reference \\
\hline 1 & ALS 2398 & $11: 35: 55$ & $-61: 36: 13$ & $2002-01-28$ & 8.39 (b) & B2 IV(pe) & 1,3 \\
\hline 5 & HD 306794 & 11:36:04 & $-61: 35: 22$ & $2002-01-28$ & 8.17 (a) & B1.5 III & 1 \\
\hline 15 & ALS 2408 & $11: 36: 31$ & $-61: 34: 25$ & 2002-01-28 & 8.51 (b) & B2 III(v) & $1,2,3$ \\
\hline 24 & CPD-603131 & $11: 36: 12$ & $-61: 35: 10$ & 2002-01-28 & 11.32 (a) & $\ldots$ & \\
\hline 26 & CPD-603125 & 11:36:09 & $-61: 35: 38$ & $2002-01-30$ & 9.20 (a) & B2 IV(ne) & 1,3 \\
\hline 27 & CPD-603128 & $11: 36: 11$ & $-61: 35: 50$ & $2002-01-28$ & 8.42 (a) & B2 IV-V & 1,3 \\
\hline 30 & CPD-603135 & $11: 36: 15$ & $-61: 36: 08$ & $2002-01-30$ & 11.10 (a) & $\ldots$ & \\
\hline 55 & CPD-603138 & $11: 36: 18$ & $-61: 37: 03$ & 2002-01-30 & 11.17 (a) & $\ldots$ & \\
\hline 97 & CPD-603098 & $11: 35: 52$ & $-61: 38: 07$ & $2002-01-28$ & $9.62(1)$ & B2 V-B1.5 III & 1,2 \\
\hline 151 & CD-603626 & $11: 36: 12$ & $-61: 32: 44$ & $2002-01-28$ & 10.39 (a) & B $2.5 \mathrm{~V}$ & 2,3 \\
\hline 169 & HD 100969 & $11: 36: 41$ & $-61: 31: 37$ & 2002-01-29 & 9.15 (a) & $\mathrm{B} 2-5$ & 2 \\
\hline 209 & HD 306800 & $11: 37: 10$ & $-61: 39: 22$ & 2002-01-29 & 10.57 (a) & B9 & 2 \\
\hline 232 & HD 100943 & $11: 36: 28$ & $-61: 39: 54$ & $2002-01-30$ & $6.92(\mathrm{~b})$ & B4 Ia-B1 Iab-Ib(e) & 1,2 \\
\hline 239 & HD 306798 & 11:36:09 & $-61: 41: 41$ & 2002-01-29 & 9.15 (b) & $\mathrm{Be}$ & 2,3 \\
\hline 240 & ALS 2401 & $11: 36: 05$ & $-61: 42: 06$ & $2002-01-29$ & $9.58(\mathrm{c})$ & $\mathrm{Be}$ & 2,3 \\
\hline 243 & HD 308740 & $11: 35: 48$ & $-61: 45: 04$ & 2002-01-29 & 11.05 (b) & B5 & 2 \\
\hline 264 & HD 306657 & $11: 35: 15$ & $-61: 41: 59$ & $2002-01-29$ & $10.58(b)$ & $\mathrm{B} 8(\mathrm{Be})$ & 2,3 \\
\hline 291 & HD 306793 & $11: 35: 22$ & $-61: 32: 11$ & 2002-01-29 & 10.77 (b) & B3 V(e) & 2 \\
\hline 301 & CPD-603087 & $11: 35.60$ & $-61: 29: 00$ & 2002-01-30 & $10.91(\mathrm{~b})$ & B3 V(e) & 2 \\
\hline 316 & BF Cen & $11: 36: 17$ & $-61: 28: 01$ & $2002-01-28$ & 8.57 (b) & B2 IV-B8(A) & 1,2 \\
\hline 317 & HD 306785 & $11: 35: 48$ & $-61: 29: 19$ & 2002-01-29 & 9.67 (b) & B2 III & 2 \\
\hline 326 & HD 100840 & $11: 35: 47$ & $-61: 33: 37$ & $2002-01-28$ & 9.70 (a) & B2 III-B7 & 1,2 \\
\hline$\ldots$ & HD 306644 & $11: 34: 50$ & $-61: 27: 56$ & 2002-01-29 & $\ldots$ & B8 & 2 \\
\hline ... & HD 306778 & $11: 37: 21$ & $-61: 26: 04$ & 2002-01-30 & $9.80(2)$ & B8 & 2 \\
\hline$\ldots$ & HD 306784 & $11: 35: 54$ & $-61: 24: 51$ & $2002-01-29$ & $\ldots$ & B5 & 2 \\
\hline$\ldots$ & HD 306787 & $11: 36: 48$ & $-61: 26: 41$ & 2002-01-29 & $\ldots$ & B9 & 2 \\
\hline$\ldots$ & HD 306788 & $11: 37: 45$ & $-61: 29: 30$ & $2002-01-30$ & $10.00(2)$ & B9 & 2 \\
\hline$\ldots$ & HD 308743 & $11: 36: 04$ & $-61: 50: 34$ & $2002-01-30$ & $\ldots$ & B8 & 2 \\
\hline$\ldots$ & HD 308852 & $11: 37: 41$ & $-61: 45: 42$ & 2002-01-30 & $10.10(2)$ & B5 & 2 \\
\hline$\ldots$ & NGC 3766 MG 29 & $11: 36: 12$ & $-61: 32: 40$ & $2002-01-28$ & $\ldots$ & $\ldots$ & \\
\hline$\ldots$ & NGC 3766 MG 116 & $11: 36: 18$ & $-61: 37: 10$ & $2002-01-30$ & $\ldots$ & $\ldots$ & \\
\hline$\ldots$ & NGC 3766 MG 177 & $11: 36: 50$ & $-61: 40: 07$ & 2002-01-30 & $\ldots$ & $\ldots$ & \\
\hline
\end{tabular}

Notes. (A) Algol eclipsing binary. (Be) Be star. (e) Emission line star. (v) Eruptive variable. (pe) Sharp $\mathrm{H} \gamma$ line and incipient emission at $\mathrm{H} \beta$. (ne) Moderate emission at H $\beta$. (a) UBV CCD data taken from Moitinho et al. (1997). (b) UBV data from Ahmed (1962). (c) UBV data from Sher (1965). (1) Spectral type taken from Schild (1970). (2) Spectral type and UBV data taken from the SIMBAD database. (3) Stars with Be phenomenon (McSwain et al. 2008).

Table 2. Stars in the open cluster NGC 4755. Nomenclature according to Arp \& van Sant (1958).

\begin{tabular}{|c|c|c|c|c|c|c|c|}
\hline $\begin{array}{l}\text { ID } \\
\text { Arp }\end{array}$ & $\begin{array}{c}\text { Other } \\
\text { designation }\end{array}$ & $\begin{array}{c}\alpha \\
(\mathrm{J} 2000)\end{array}$ & $\begin{array}{c}\delta \\
(\mathrm{J} 2000)\end{array}$ & $\begin{array}{c}\text { Observation } \\
\text { date }\end{array}$ & $m_{\mathrm{v}}$ & S.T & Reference \\
\hline 1 & NSV 6008 & $12: 53: 21.90$ & $-60: 19: 42.55$ & $2003-06-03$ & 5.75 & B9 Ia, B9 Ia-Iab (vs) & $1,2,3,4,5$ \\
\hline 5 & CPD-594552 & $12: 53: 46.49$ & $-60: 24: 12.42$ & 2003-06-03 & 8.35 & B1 III & $3,4,5$ \\
\hline 6 & ALS 2816 & $12: 53: 57.54$ & $-60: 24: 58.08$ & 2003-06-05 & 9.09 & B0.5-1 II-III, B2 III, B1 V (vc) & $2,3,4,5$ \\
\hline 7 & BS Cru & $12: 53: 20.67$ & $-60: 23: 16.90$ & 2003-06-04 & 9.79 & $\mathrm{~B} 0.5 \mathrm{~V}, \mathrm{~B} 1 \mathrm{~V}(\mathrm{vc})$ & 3,5 \\
\hline 8 & CPD-594540 & $12: 53: 33.19$ & $-60: 24: 33.30$ & 2003-06-05 & 9.93 & B $1.5 \mathrm{~V}$ & 5 \\
\hline 11 & CPD-594530 & $12: 53: 22.58$ & $-60: 23: 47.70$ & 2003-06-05 & 11.42 & B8 V, B3 V & 3,5 \\
\hline 106 & BU Cru & $12: 53: 37.62$ & $-60: 21: 25.42$ & 2003-06-04 & 7.00 & B2 Ib, B1.5 Ib & $2,3,4,5$ \\
\hline 113 & CPD-594532 & $12: 53: 25.66$ & $-60: 22: 00.20$ & 2003-06-04 & 10.23 & B1 V (vc) & 3 \\
\hline 117 & CPD-594531 & $12: 53: 24.25$ & $-60: 21: 30.90$ & 2003-06-05 & 10.87 & Bnn, B2.5 Vn & 3,5 \\
\hline 201 & EI Cru & $12: 53: 52.00$ & $-60: 22: 15.90$ & 2003-06-04 & 9.37 & B1 V, B0.5 V (vc) & $3,4,5$ \\
\hline 202 & CX Cru & $12: 53: 51.73$ & $-60: 21: 58.60$ & 2003-06-04 & 10.06 & $\mathrm{~B} 1 \mathrm{~V}(\mathrm{vc})$ & 3 \\
\hline 223 & CC Cru & $12: 53: 47.28$ & $-60: 19: 55.32$ & 2003-06-03 & 7.93 & B0 V?, B0.5 Vn, B2 III (ve) & $3,4,5$ \\
\hline 305 & $\mathrm{CN}$ Cru & $12: 53: 49.50$ & $-60: 23: 02.70$ & 2003-06-05 & 8.43 & B2, B0.5 V, B0.5 IVn, B1 V (eb) & $2,3,4,5$ \\
\hline 306 & CPD-594559 & $12: 53: 51.57$ & $-60: 23: 16.90$ & 2003-06-05 & 9.98 & Bnne $(\mathrm{Be})$ & 3 \\
\hline 418 & CPD-594542 & $12: 53: 35.49$ & $-60: 23: 46.70$ & 2003-06-03 & 9.86 & B2 V, B1.5 V (vs) & 3,5 \\
\hline 452 & HD 312079 & $12: 53: 10.22$ & $-60: 25: 59.60$ & 2003-06-03 & 10.12 & $\mathrm{~B} 1.5 \mathrm{~V}$ & 5 \\
\hline 454 & HD 312080 & 12:53:14.26 & $-60: 27: 38.07$ & 2003-06-03 & 10.11 & B2 V & 5 \\
\hline
\end{tabular}

Notes. (Be) Be star. (vs) Variable star. (vc) Variable star of beta Cep type. (ve) Ellipsoidal variable star. (eb) Eclipsing binary of beta Lyr type. $m_{v}$ taken from Arp \& van Sant (1958). (1) Spectral types taken from Bidelman (1954). (2) Spectral types taken from Hernández (1960). (3) Spectral types taken from Feast (1963). (4) Spectral types taken from Schild (1970). (5) Spectral types taken from Evans et al. (2005) and Dufton et al. (2006). 
and 5) it can be applied with high accuracy to normal and many peculiar classes of B-type stars (Zorec \& Briot 1991; Cidale et al. 2001; Zorec et al. 2005; Cidale et al. 2007), even if these objects display a second BD that could be either in emission or in absorption (Divan 1979).

To determine the parameter $D=\log \left(F_{+3700} / F_{-3700}\right)$ we normalized the observed energy distribution with a Planck function to rectify the energy distribution at both sides of the BD. Then, we extrapolated the Paschen continuum to obtain the flux at $F_{+3700}$. To determine $F_{-3700}$, we used the flux level where the higher members of the Balmer lines are merged. Using Balmer lines to determine the low level of the Balmer jump has the advantage of giving an estimate of the BD that is barely or not at all affected by possible variations of the intensity of the Balmer continuum.

The BCD system also enables one to derive the stellar color excess produced by the ISM and circumstellar extinctions. This parameter can be determined through the color gradient $\Phi_{\mathrm{ab}}$, which is defined by Allen (1976) as

$\Phi_{\mathrm{ab}}=-\ln \left[\frac{\lambda_{\mathrm{a}}^{5} F_{\lambda_{\mathrm{a}}}}{\lambda_{\mathrm{b}}^{5} F_{\lambda_{\mathrm{b}}}}\right] /\left(\frac{1}{\lambda_{\mathrm{a}}}-\frac{1}{\lambda_{\mathrm{b}}}\right)$,

where $\lambda_{\mathrm{a}}$ and $\lambda_{\mathrm{b}}$ are the wavelength values at the endpoints of a given interval.

According to the empirical relation between the color excess and the color gradient derived by Chalonge \& Divan (1973), the visual absorption coefficient can be expressed as

$A_{\mathrm{v}}=3.1 E(B-V)=1.7\left(\Phi_{\mathrm{rb}}-\Phi_{\mathrm{rb}}^{0}\right)=1.9\left(\Phi_{\mathrm{b}}-\Phi_{\mathrm{b}}^{0}\right) \mathrm{mag}$,

where $\Phi_{\mathrm{rb}}$ and $\Phi_{\mathrm{rb}}^{0}$ are the observed and intrinsic color gradients defined in the spectral region $4000-6700 \AA$, and $\Phi_{\mathrm{b}}$ and $\Phi_{\mathrm{b}}^{0}$ are those defined in 4000-4800 $\AA$.

In the same way, in the Balmer continuum region ( $\lambda \lambda 3150-3700 \AA)$ the color gradient $\Phi_{\mathrm{uv}}$ is defined. Often this color gradient is not used when the star is a Be star either in the Be or in the Be-shell phase.

In Fig. 3 we show the behavior of the Balmer continuum at $\lambda<3700 \AA$ and describe details on the tracing of the rectified Paschen continuum spectrum in three cases:

a) a normal B-type star;

b) a Be star with a second component of the BD in emission (flux excess as compared to a normal flux level), for instance, during a phase of strong Balmer line emission phase; and

c) a Be star with a second component of the BD in strong absorption (flux deficiency). This is the case of a star in a Be-shell phase.

The BCD parameters $\left(D, \lambda_{1}, \Phi\right)$ were measured with an interactive code developed by one of us (Y.A.) and the obtained results are shown in Sect. 4.

\subsubsection{New relationship between $E(B-V)$ and the color gradient excess}

Because our observed wavelength range is constrained to the interval 3500-4700 $\AA$, we searched for a new linear relation between the reddening and color gradient excess. To carry out this task, we used theoretical spectra computed by Kurucz (1979), which were reddened assuming $E(B-V)$ values from 0 to 0.5 with step 0.1 . We adopted the ISM extinction law given by Savage \& Mathis (1979). The theoretical relation is shown in

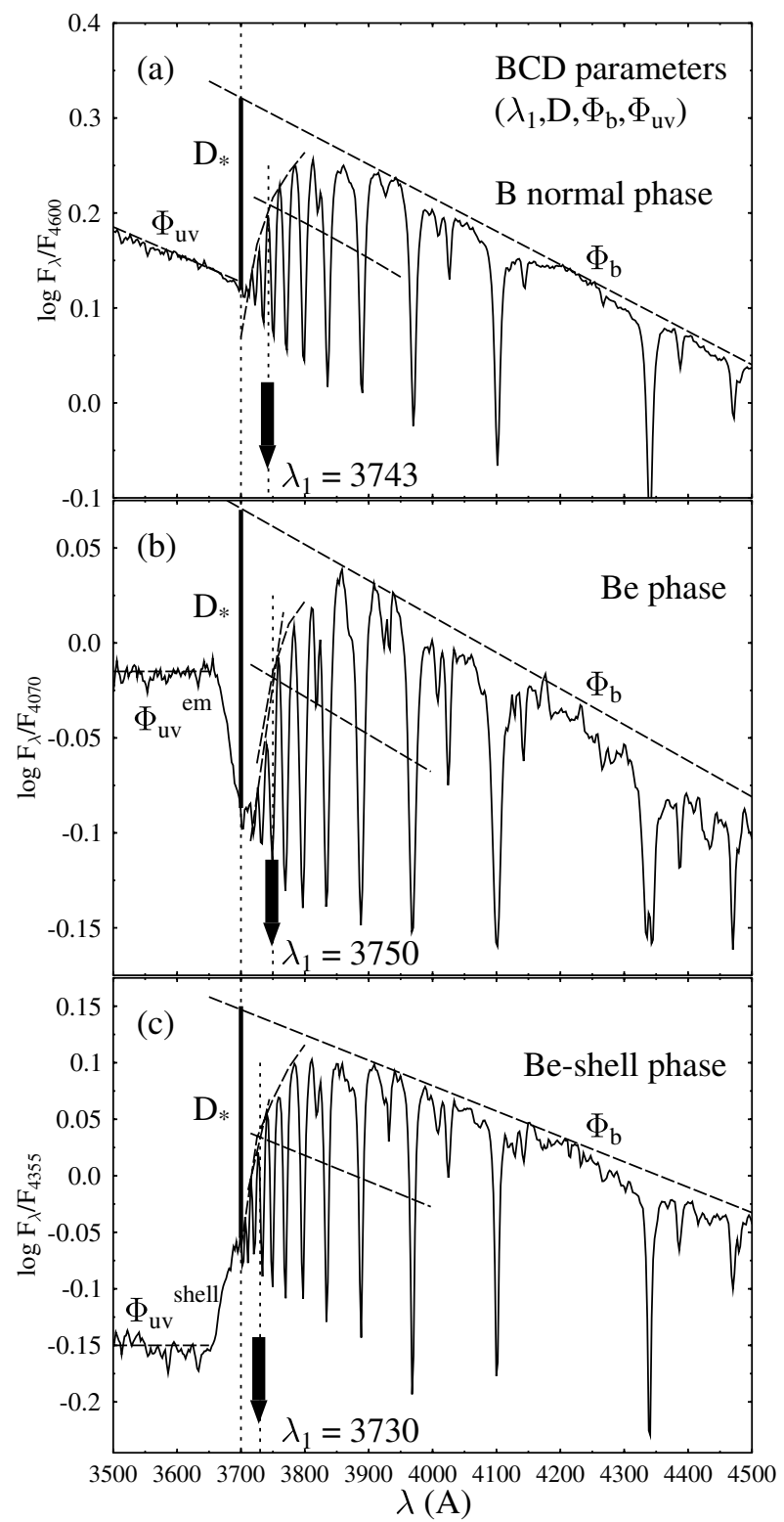

Fig. 3. Graphical explanation of the BCD spectrophotometric parameters $\left(D, \lambda_{1}, \Phi_{\mathrm{b}}, \Phi_{\mathrm{uv}}\right)$. In a) the determination of the BCD parameters is shown for a star in a B-normal type phase; b) displays a Be star whose second component of the Balmer discontinuity is in emission (flux excess); c) is for a Be-shell phase where the second component of the Balmer discontinuity appears in absorption (flux deficiency). The parameter $D_{*}$ (heavy full line) that measures the photospheric component of the Balmer discontinuity is given in dex, while the average spectral position of the Balmer discontinuity is generally given in $\lambda_{1}-3700 \AA$. In stars with a second component in emission or in absorption the parameter $\Phi_{\mathrm{uv}}$ is generally not measured.

Fig. 4 and the best fit was obtained using square minima. The relation between $A_{\mathrm{v}}$ and the theoretical $\Phi_{\mathrm{bb}}$ gradient excess is

$A_{\mathrm{v}}=3.1 E(B-V)=2.3\left(\Phi_{\mathrm{bb}}-\Phi_{\mathrm{bb}}^{0}\right) \mathrm{mag}$,

where $\Phi_{\mathrm{bb}}$ and $\Phi_{\mathrm{bb}}^{0}$ are the observed and intrinsic color gradients in the spectral range 4000-4600 ̊.

We also computed the color gradient excesses, $\Phi_{\mathrm{b}}$ and $\Phi_{\mathrm{rb}}$ in the same way. The theoretical relations are

$A_{\mathrm{v}}=3.1 E(B-V)=1.7\left(\Phi_{\mathrm{rb}}-\Phi_{\mathrm{rb}}^{0}\right)=2.1\left(\Phi_{\mathrm{b}}-\Phi_{\mathrm{b}}^{0}\right) \mathrm{mag}$, 


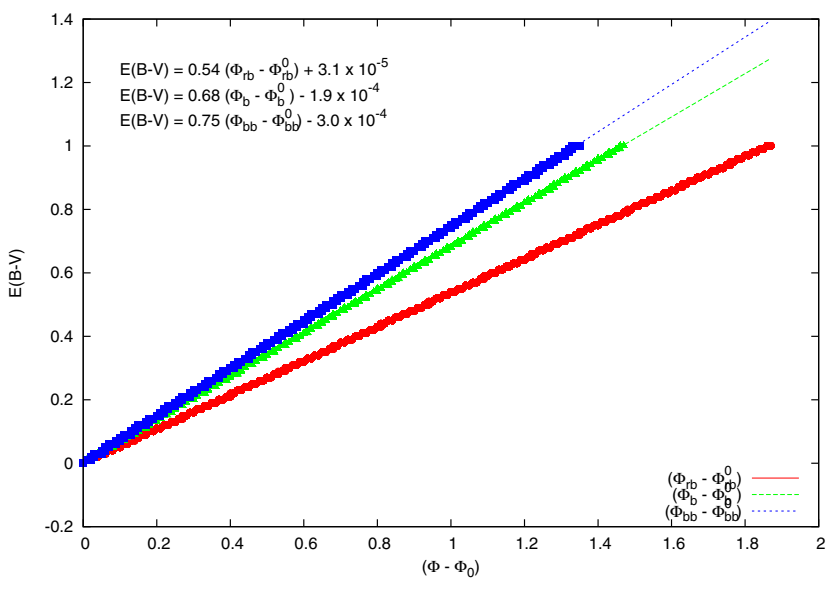

Fig. 4. Theoretical relation between $E(B-V)$ and the color gradient excess for different wavelength ranges.

the coefficient obtained for $\Phi_{\mathrm{rb}}$ agrees excellently with that shown in Eq. (2). However, the theoretical coefficient obtained in the wavelength interval 4000-4800 $\AA$, which corresponds to the color gradient $\Phi_{\mathrm{b}}$, is slightly larger. This difference can simply be due to the standard ISM extinction law used here (Savage \& Mathis 1979) and by Divan (1954).

Another alternative method is to derive the color excess using the photometric $B-V$ values, available in the literature, and the intrinsic color $(B-V)_{0}$, obtained from the measured BCD $T_{\text {eff }}$ values together with the $T_{\text {eff }}$-color calibration given by Flower (1996).

\subsection{Fundamental parameters of the cluster}

The BCD spectrophotometric system enables us to derive absolute magnitudes $M_{\mathrm{v}}$ and $M_{\mathrm{bol}}$, and the color excess, $E(B-V)$, for each star. These quantities, together with the photometric apparent magnitudes, $m_{\mathrm{v}}$, give an estimate of the distance modulus corrected for interstellar extinction using the well-known Pogson relation:

$\left(m_{\mathrm{v}}-M_{\mathrm{v}}\right)_{0}=m_{\mathrm{v}}-M_{\mathrm{v}}-A_{\mathrm{v}}=-5+5 \log d$,

where $A_{\mathrm{v}}=R_{\mathrm{v}} E(B-V)$. We adopted a standard selective absorption coefficient, $R_{\mathrm{v}}=3.1$ (Schultz \& Wiemer 1975; Whittet \& van Breda 1980; Rieke \& Lebofsky 1985).

The distance modulus of a cluster is the average value of all individual member determinations. As a criterion, we considered that stars with a distance modulus $3 \sigma$ larger than the obtained mean value are probably non-members (pnm).

To derive the age of the cluster we adopted the isochrones given by Bressan et al. (1993). Because the stellar sample of each cluster is mostly determined by early-type stars, we also used parameters of evolved stars previously studied in the literature.

\section{Results}

\subsection{Fundamental parameters of the stars}

Applying the BCD system, we measured $D$ and $\lambda_{1}$ for the program stars of NGC 3766 and NGC 4755. We determined their spectral type, $T_{\text {eff }}, \log g$, and absolute magnitudes $M_{\mathrm{v}}$ and $M_{\mathrm{bol}}$ (see Tables 3 and 4 ) using the respective calibrations of $\left(\lambda_{1}, D\right)$ given by Divan \& Zorec (1982), Zorec (1986), Zorec \& Briot (1991), and Zorec et al. (2009). The uncertainties on $T_{\text {eff }}, \log g$, $M_{\mathrm{v}}$, and $M_{\mathrm{bol}}$ are mainly related to the errors of the BCD observational quantities $\left(\lambda_{1}, D\right)$. Typical values are $\sigma(D) \lesssim 0.015 \mathrm{dex}$ and $\sigma\left(\lambda_{1}\right) \sim 5 \AA$, which produce $\Delta T_{\text {eff }} \sim \pm 500 \mathrm{~K}$ for the late B-type stars and $\pm 1500 \mathrm{~K}$ for the early B-types $\left(T_{\text {eff }}>\right.$ $20000 \mathrm{~K}), \Delta \log g \sim \pm 0.2 \mathrm{dex}$, and $\Delta M \sim \pm 0.3 \mathrm{mag}$ both for $M_{\mathrm{V}}$ and $M_{\text {bol }}$.

We also determined the color excesses, $E(B-V)$ and derived the distance moduli. In addition, we measured the rotation velocity of the stars from each spectrum. This was obtained from the FWHM of $\mathrm{He}_{\mathrm{I}}$ and $\mathrm{Mg}$ II lines. To estimate the rotation velocity we used our line FWHM measurements corresponding to 20 stars that had previous $V \sin i$ determinations by Slettebak (1985), Głębocki \& Gnaciński (2005), and Huang \& Gies (2006). From the linear relation in the velocity range between 20 and $300 \mathrm{~km} \mathrm{~s}^{-1}$, we derived the $V \sin i$ of the whole sample. These data were also included in Tables 3 and 4 . Because we used low-resolution spectra, uncertainties in the rotation velocities of about $50 \mathrm{~km} \mathrm{~s}^{-1}$ are expected.

In addition, with the BCD $T_{\text {eff }}$ and $M_{\text {bol }}$ values listed in Tables 3 and 4, we computed the luminosity of the stars relative to the Sun's luminosity $\left(\log L / L_{\odot}\right)$ and interpolated in the evolutionary diagrams given by Ekström et al. (2012) to obtain masses, ages, radii, and $\log g$. These values, derived from evolutionary models without rotation and for metallicity $Z=$ 0.014 , are given in Tables 5 and 6 . The same parameters but derived from evolutionary models with rotation and metallicity $Z=0.014$ are given in Tables 7 and 8 . We note that the models with rotation are calculated for an initial ratio of angular velocities $\Omega / \Omega_{\mathrm{c}}=0.5$. Because each star can have a different angular velocity ratio in the ZAMS, differences can exist in the estimate of its fundamental parameters. These models give an order of magnitude of the effects induced by the stellar rotation, however. We point out though that we used the apparent $T_{\text {eff }}$ and $M_{\text {bol }}$ in both cases to interpolate masses and ages in the evolutionary tracks. A consistent treatment of the fundamental parameters would still need to include the effects the rotation has on the observed fundamental parameters, as in Frémat et al. (2005) and Zorec et al. (2005), but such corrections are far beyond the scope of this paper.

\subsection{Fundamental parameters of the clusters}

\subsubsection{NGC 3766}

NGC $3766\left(\alpha=11^{\mathrm{h}} 36^{\mathrm{m}} 13^{\mathrm{s}} .3\right.$ and $\left.\delta=-61^{\circ} 36^{\prime} 55^{\prime \prime}\right)$ is a young open cluster in the Carina spiral arm, which is rich in B and Be stars (Slettebak 1985). Photometric and spectroscopic studies of this cluster were performed by Ahmed (1962), Schild (1970), Harris (1976), Yilmaz (1976), Mermilliod (1982), Shobbrook (1985), Slettebak (1985), Shobbrook (1987), Moitinho et al. (1997), Piatti et al. (1998), Tadross (2001), Levesque et al. (2005), McSwain \& Gies (2005) and McSwain et al. (2008). However, the cluster's distance (between 1.5 and $2.2 \mathrm{kpc}$ ) and age (from 14.5 to $25 \mathrm{Myr}$ ) remain somewhat uncertain. The reddening $E(B-V)$ of the cluster is between 0.16 and $0.22 \mathrm{mag}$. The $E(B-V)$ values in some cases could be misestimated since the intrinsic colors were obtained using spectral types and luminosity classes of variables or Be stars available in the literature (Moitinho et al. 1997).

To discuss the specific characteristics of this cluster, we used the procedure described in Sect. 3. We find that the cluster shows a high inhomogeneous reddening with a mean color excess $\overline{E(B-V)}=0.21$. We estimated a mean distance modulus $\overline{\left(m_{\mathrm{v}}-M_{\mathrm{v}}\right)_{0}}=11.57 \pm 0.42$ taking into account the entire sample. However, if we reject the stars that show discrepancies greater 
Table 3. NGC 3766: stellar fundamental parameters of the BCD system.

\begin{tabular}{|c|c|c|c|c|c|c|c|c|c|c|c|}
\hline $\begin{array}{l}\text { ID } \\
\text { Ahmed }\end{array}$ & $\begin{array}{c}\text { Other } \\
\text { designation }\end{array}$ & $\begin{array}{c}D \\
{[\mathrm{dex}]}\end{array}$ & $\begin{array}{c}\lambda_{1} \\
{[\AA]}\end{array}$ & S.T & $\begin{array}{l}T_{\text {eff }} \\
{[\mathrm{K}]}\end{array}$ & $\begin{array}{l}\log g \\
\text { [dex] }\end{array}$ & $\begin{array}{c}M_{\mathrm{v}} \\
{[\mathrm{mag}]}\end{array}$ & $\begin{array}{c}M_{\mathrm{bol}} \\
{[\mathrm{mag}]}\end{array}$ & $\begin{array}{c}E(B-V) \\
{[\mathrm{mag}]}\end{array}$ & $\begin{array}{c}\left(m_{\mathrm{v}}-M_{\mathrm{v}}\right)_{0} \\
{[\mathrm{mag}]}\end{array}$ & $\begin{array}{c}V \sin (i) \\
{\left[\mathrm{km} \mathrm{s}^{-1}\right]}\end{array}$ \\
\hline 1 & ALS 2398 & 0.203 & 32.07 & B5 III & 16170 & 2.81 & -4.05 & -4.55 & 0.23 & 11.97 & 110 \\
\hline 5 & HD 306794 & 0.110 & 41.75 & B2 III & 22780 & 3.06 & -4.56 & -6.09 & 0.23 & 12.02 & 000 \\
\hline 15 & ALS 2408 & 0.190 & 35.08 & B4 III & 17660 & 2.80 & -4.09 & -4.51 & 0.29 & 11.72 & 110 \\
\hline 24 & CPD-603131 & 0.278 & 60.07 & B5 V & 15110 & 4.24 & -0.02 & -1.58 & 0.25 & 10.54 & 370 \\
\hline 26 & CPD-603125 & 0.172 & 49.45 & B2 IV & 21040 & 3.81 & -2.51 & -4.01 & 0.00 & 11.57 & 370 \\
\hline 27 & CPD-603128 & 0.202 & 45.05 & B3 III & 20580 & 3.49 & -2.58 & -3.59 & 0.25 & $10.28^{p n m}$ & 180 \\
\hline 30 & CPD-603135 & 0.249 & 54.43 & $\mathrm{~B} 4 \mathrm{~V}$ & 18080 & 4.11 & -1.08 & -2.20 & 0.07 & 11.97 & 300 \\
\hline 55 & CPD-603138 & 0.356 & 49.78 & B7 IV & 12590 & 4.02 & -0.54 & -1.03 & 0.28 & $10.01^{p n m}$ & $\ldots$ \\
\hline 97 & CPD-60 3098 & 0.193 & 46.92 & B3 IV & 20250 & 3.62 & -2.59 & -3.68 & 0.16 & 11.70 & 60 \\
\hline 151 & CD-603626 & 0.224 & 52.17 & B3 V & 17950 & 4.01 & -1.56 & -3.13 & 0.13 & 10.56 & 200 \\
\hline 169 & HD 100969 & 0.148 & 37.06 & B2 II & 20480 & 2.80 & -4.60 & -5.23 & 0.27 & 12.79 & 165 \\
\hline 209 & HD 306800 & 0.206 & 54.51 & B3 V & 20600 & 4.03 & -1.65 & -3.04 & 0.37 & 11.31 & 270 \\
\hline 232 & HD $100943^{d d}$ & 0.105 & 19.02 & B3 Ia & 15680 & $<2.80$ & $-7.00^{B}$ & $-8.37^{F}$ & 0.21 & $13.50^{p n m}$ & 75 \\
\hline 239 & HD 306798 & 0.182 & 46.84 & B3 IV & 20300 & 3.62 & -2.53 & -4.07 & 0.37 & 10.76 & 120 \\
\hline 240 & ALS $2401^{d d}$ & 0.217 & 30.94 & B5 II & 15380 & $\sim 2.80$ & -4.01 & -4.57 & 0.31 & 12.65 & 215 \\
\hline 243 & HD 308740 & 0.245 & 61.60 & B5 V & 17970 & 4.24 & -1.11 & -2.09 & 0.42 & 10.91 & 110 \\
\hline 264 & HD $306657^{d d}$ & 0.150 & 57.66 & $\mathrm{~B} 2 \mathrm{~V}$ & 25740 & 4.04 & -2.62 & -4.13 & 0.43 & 11.78 & 170 \\
\hline 291 & HD 306793 & 0.216 & 54.36 & B3 V & 18490 & 4.04 & -1.53 & -3.10 & 0.34 & 11.14 & 360 \\
\hline 301 & CPD-60 3087 & 0.221 & 52.68 & B3 V & 18150 & 4.01 & -1.57 & -3.12 & 0.00 & 12.47 & 335 \\
\hline 316 & BF Cen & 0.138 & 46.60 & B2 III & 22580 & 3.45 & -3.60 & -5.08 & 0.22 & 11.73 & 175 \\
\hline 317 & HD 306785 & 0.177 & 47.10 & B2 III & 20580 & 3.62 & -2.59 & -4.00 & 0.31 & 11.35 & 350 \\
\hline 326 & HD 100840 & 0.179 & 32.86 & B4 II & 17740 & $<2.80$ & -4.61 & -5.01 & 0.28 & 11.94 & 90 \\
\hline$\cdots$ & HD 306644 & 0.314 & 36.53 & B6 III & 15660 & 3.26 & -2.10 & -2.53 & 0.38 & $\ldots$ & 115 \\
\hline$\cdots$ & HD 306778 & 0.195 & 53.87 & B3 V & 20310 & 4.00 & -2.10 & -3.61 & 0.00 & 11.90 & 140 \\
\hline$\cdots$ & HD 306784 & 0.341 & 48.53 & B7 IV & 13150 & 4.03 & -0.01 & -1.17 & 0.22 & $\ldots$ & $\ldots$ \\
\hline$\cdots$ & HD 306787 & 0.257 & 55.47 & B5 V & 16220 & 4.11 & -1.06 & -2.03 & 0.35 & $\ldots$ & 265 \\
\hline$\cdots$ & HD 306788 & 0.191 & 53.50 & B3 V & 20090 & 4.01 & -2.06 & -3.57 & 0.00 & 12.06 & 130 \\
\hline$\cdots$ & HD 308743 & 0.227 & 52.99 & B3 V & 17780 & 4.01 & -1.52 & -2.66 & 0.07 & $\ldots$ & 430 \\
\hline$\ldots$ & HD $308852^{d d}$ & 0.273 & 45.36 & B5 IV & 15570 & 3.83 & -1.54 & -2.21 & 0.14 & 11.19 & $\ldots$ \\
\hline$\ldots$ & NGC 3766 MG 29 & 0.221 & 55.77 & B3 V & 18210 & 4.11 & -1.58 & -2.70 & 0.08 & $\ldots$ & 415 \\
\hline .. & NGC 3766 MG 116 & 0.245 & 60.61 & B5 V & 17980 & 4.22 & -1.10 & -2.12 & 0.15 & 10.93 & $\ldots$ \\
\hline$\ldots$ & NGC 3766 MG 177 & 0.179 & 47.32 & B3 IV & 20450 & 3.60 & -2.53 & -4.04 & 0.00 & $\ldots$ & 190 \\
\hline
\end{tabular}

Notes. $g$ stellar surface gravity given in $\mathrm{cm} \mathrm{s}^{-2}$. ${ }^{(d d)}$ Stars with double Balmer discontinuity. ${ }^{(B)}$ Values of $M_{\mathrm{v}}$ were adopted from Balona \& Crampton (1974). ${ }^{(F)} M_{\text {bol }}$ values were calculated using the bolometric correction given by Flower (1996). ${ }^{(p n m)}$ Possible non-member stars.

Table 4. NGC 4755: stellar fundamental parameters of the BCD system.

\begin{tabular}{lccccccccccc}
\hline \hline $\begin{array}{l}\text { ID } \\
\text { Arp }\end{array}$ & $\begin{array}{c}\text { Other } \\
\text { designation }\end{array}$ & $\begin{array}{c}D \\
{[\mathrm{dex}]}\end{array}$ & $\begin{array}{c}\lambda_{1} \\
{[\AA]}\end{array}$ & S.T & $\begin{array}{c}T_{\text {eff }} \\
{[\mathrm{K}]}\end{array}$ & $\begin{array}{c}\log g \\
{[\mathrm{dex}]}\end{array}$ & $\begin{array}{c}M_{\mathrm{v}} \\
{[\mathrm{mag}]}\end{array}$ & $\begin{array}{c}M_{\mathrm{bol}} \\
{[\mathrm{mag}]}\end{array}$ & $\begin{array}{c}E(B-V) \\
{[\mathrm{mag}]}\end{array}$ & $\begin{array}{c}\left(m_{\mathrm{v}}-M_{\mathrm{v}}\right)_{0} \\
{\left[\mathrm{mag}^{2}\right.}\end{array}$ & $\begin{array}{c}V \text { sin }(i) \\
{\left[\mathrm{km} \mathrm{s}{ }^{-1}\right]}\end{array}$ \\
\hline 1 & NSV 6008 & 0.141 & 9.75 & B7 Ia & 12680 & $<2.80$ & $-7.00^{B}$ & $-7.82^{F}$ & 0.38 & 11.59 & 80 \\
5 & CPD-594552 & 0.098 & 29.14 & B2 I & 20710 & $<2.80$ & $-7.00^{B}$ & -7.63 & 0.16 & $14.79^{\text {pnm }}$ & 70 \\
6 & ALS 2 816 & 0.116 & 47.63 & B1 III & 25980 & 3.25 & -3.70 & -5.56 & 0.39 & 11.51 & 55 \\
7 & BS Cru & 0.111 & 44.57 & B1 III & 23610 & 3.02 & -4.17 & -5.68 & 0.17 & $13.39^{p n m}$ & $\ldots$ \\
8 & CPD-594540 & 0.190 & 60.52 & B3 V & 20220 & 4.22 & -1.54 & -3.16 & 0.36 & $10.31^{p n m}$ & 185 \\
11 & CPD-594530 & 0.291 & 65.23 & B6 V & 15620 & $>4.30$ & -0.64 & -1.60 & 0.44 & $10.66^{p n m}$ & 290 \\
106 & BU Cru & 0.078 & 26.45 & B2 Ia & 20620 & $<2.80$ & $-7.00^{B}$ & $-9.84^{F}$ & 0.29 & 13.02 & $\ldots$ \\
113 & CPD-594532 & 0.144 & 53.74 & B2 IV & 25890 & 3.89 & -2.67 & -4.58 & 0.22 & 12.14 & $\ldots$ \\
117 & CPD-594531 & 0.189 & 62.00 & B3 V & 20240 & 4.21 & -1.52 & -3.13 & 0.39 & 11.20 & 400 \\
201 & EI Cru & 0.122 & 47.11 & B1 III & 23730 & 3.26 & -3.62 & -5.62 & 0.18 & 12.45 & $\ldots$ \\
202 & CX Cru & 0.131 & 51.61 & B2 IV & 25820 & 3.81 & -3.07 & -5.09 & 0.22 & 12.40 & $\ldots$ \\
223 & CC Cru & 0.099 & 28.03 & B2 Ia & 18710 & $<2.80$ & $-7.00^{B}$ & -7.58 & 0.30 & $13.91^{p n m}$ & 125 \\
305 & CN Cru & 0.108 & 61.49 & B0 V & 31170 & 4.12 & -3.07 & -5.15 & 0.43 & $10.32^{p n m}$ & 165 \\
306 & CPD-594559dd & 0.079 & 51.63 & B0 III & 30040 & 3.44 & -4.22 & -6.59 & 0.62 & 12.29 & 430 \\
418 & CPD-594542 & 0.118 & 44.11 & B2 III & 23070 & 3.02 & -4.05 & -5.51 & 0.17 & 13.19 & 180 \\
452 & HD 312079 & 0.166 & 54.87 & B2 V & 22860 & 4.01 & -2.22 & -4.08 & 0.25 & 11.49 & 135 \\
454 & HD 312 080 & 0.163 & 51.15 & B2 IV & 22990 & 3.83 & -2.52 & -4.09 & 0.20 & 11.86 & 100 \\
\hline
\end{tabular}

Notes. ${ }^{(d d)}$ Stars with double Balmer discontinuity. ${ }^{(B)}$ Values of $M_{\mathrm{v}}$ were adopted from Balona \& Crampton $(1974) .{ }^{(F)} M_{\text {bol }}$ values were calculated using the bolometric corrections given by Flower (1996). ${ }^{\left({ }^{p n m}\right)}$ Possible non-member stars. 
Table 5. NGC 3766: data obtained from interpolations in evolutionary diagrams given by Ekström et al. (2012) without rotation.

\begin{tabular}{|c|c|c|c|c|c|c|c|c|}
\hline $\begin{array}{l}\text { ID } \\
\text { Ahmed }\end{array}$ & $\begin{array}{c}\text { Other } \\
\text { designation }\end{array}$ & $\begin{array}{c}\log T_{\text {eff }} \\
{[\mathrm{K}]}\end{array}$ & $\log L / L_{\odot}$ & $M / M_{\odot}$ & $R / R_{\odot}$ & $\log g$ & $\begin{array}{c}\text { age } \\
{\left[10^{6} \mathrm{yr}\right]}\end{array}$ & $\log g_{\mathrm{BCD}}$ \\
\hline 1 & ALS 2398 & 4.209 & 4.03 & $9.3 \pm 0.2$ & $13.3 \pm 1.3$ & $3.16 \pm 0.09$ & $24.9 \pm 1.1$ & 2.81 \\
\hline 5 & HD 306794 & 4.358 & 4.58 & $11.9 \pm 2.6$ & $12.6 \pm 1.2$ & $3.31 \pm 0.18$ & $16.8 \pm 5.4$ & 3.06 \\
\hline 15 & ALS 2408 & 4.247 & 4.12 & $9.9 \pm 0.2$ & $12.3 \pm 1.2$ & $3.25 \pm 0.09$ & $22.0 \pm 0.9$ & 2.80 \\
\hline 24 & CPD-603131 & 4.179 & 2.37 & $2.1 \pm 1.3$ & $2.2 \pm 0.2$ & $4.07 \pm 0.35$ & $10.7 \pm 15.8$ & 4.24 \\
\hline 26 & CPD-603125 & 4.323 & 3.67 & $8.3 \pm 0.2$ & $5.2 \pm 0.5$ & $3.93 \pm 0.09$ & $23.8 \pm 3.3$ & 3.81 \\
\hline 27 & CPD-603128 & 4.313 & 3.67 & $8.2 \pm 0.2$ & $5.4 \pm 0.5$ & $3.88 \pm 0.09$ & $25.6 \pm 3.0$ & 3.49 \\
\hline 30 & CPD-603135 & 4.257 & 2.94 & $4.0 \pm 2.0$ & $3.0 \pm 0.3$ & $4.08 \pm 0.30$ & $18.9 \pm 15.7$ & 4.11 \\
\hline 55 & CPD-603138 & 4.100 & 2.46 & $3.8 \pm 0.1$ & $3.6 \pm 0.3$ & $3.90 \pm 0.09$ & $143.0 \pm 15.4$ & 4.02 \\
\hline 97 & CPD-603098 & 4.306 & 3.66 & $8.1 \pm 0.2$ & $5.5 \pm 0.5$ & $3.86 \pm 0.09$ & $26.9 \pm 3.0$ & 3.62 \\
\hline 151 & CD-603626 & 4.254 & 3.12 & $6.0 \pm 0.1$ & $3.8 \pm 0.4$ & $4.06 \pm 0.09$ & $38.3 \pm 8.9$ & 4.01 \\
\hline 169 & HD 100969 & 4.311 & 4.48 & $12.1 \pm 0.6$ & $13.8 \pm 1.3$ & $3.24 \pm 0.10$ & $15.7 \pm 1.8$ & 2.80 \\
\hline 209 & HD 306800 & 4.314 & 3.30 & $6.9 \pm 0.2$ & $3.5 \pm 0.3$ & $4.18 \pm 0.10$ & $13.4 \pm 10.0$ & 4.03 \\
\hline 232 & HD 100943 & 4.195 & 5.19 & $21.8 \pm 0.6$ & $53.4 \pm 5.2$ & $2.32 \pm 0.10$ & $7.0 \pm 0.2$ & 2.80 \\
\hline 239 & HD 306798 & 4.307 & 3.64 & $8.0 \pm 0.2$ & $5.4 \pm 0.5$ & $3.88 \pm 0.09$ & $26.8 \pm 3.2$ & 3.62 \\
\hline 240 & ALS 2401 & 4.187 & 3.98 & $9.0 \pm 0.2$ & $13.8 \pm 1.3$ & $3.11 \pm 0.09$ & $26.7 \pm 1.1$ & 2.80 \\
\hline 243 & HD 308740 & 4.255 & 2.94 & $5.5 \pm 0.2$ & $3.1 \pm 0.3$ & $4.20 \pm 0.10$ & $22.1 \pm 16.3$ & 4.24 \\
\hline 264 & HD 306657 & 4.411 & 3.95 & $10.8 \pm 0.4$ & $4.8 \pm 0.5$ & $4.11 \pm 0.10$ & $9.1 \pm 3.8$ & 4.04 \\
\hline 291 & HD 306793 & 4.267 & 3.14 & $6.1 \pm 0.2$ & $3.6 \pm 0.3$ & $4.10 \pm 0.10$ & $31.9 \pm 9.8$ & 4.04 \\
\hline 301 & CPD-60 3087 & 4.259 & 3.14 & $6.0 \pm 0.1$ & $3.8 \pm 0.4$ & $4.06 \pm 0.09$ & $36.4 \pm 8.8$ & 4.01 \\
\hline 316 & BF Cen & 4.354 & 4.19 & $11.2 \pm 0.2$ & $8.2 \pm 0.8$ & $3.66 \pm 0.09$ & $16.2 \pm 1.3$ & 3.45 \\
\hline 317 & HD 306785 & 4.313 & 3.68 & $8.2 \pm 0.2$ & $5.5 \pm 0.5$ & $3.88 \pm 0.09$ & $25.6 \pm 3.0$ & 3.62 \\
\hline 326 & HD 100840 & 4.249 & 4.33 & $11.5 \pm 0.2$ & $15.6 \pm 1.5$ & $3.11 \pm 0.09$ & $16.7 \pm 0.6$ & 2.80 \\
\hline$\cdots$ & HD 306644 & 4.195 & 3.22 & $5.9 \pm 0.1$ & $5.6 \pm 0.5$ & $3.71 \pm 0.09$ & $59.3 \pm 5.3$ & 3.26 \\
\hline$\cdots$ & HD 306778 & 4.308 & 3.47 & $7.4 \pm 0.2$ & $4.4 \pm 0.4$ & $4.02 \pm 0.09$ & $25.4 \pm 5.3$ & 4.00 \\
\hline$\cdots$ & HD 306784 & 4.119 & 2.28 & $3.6 \pm 0.1$ & $2.7 \pm 0.2$ & $4.14 \pm 0.10$ & $109.0 \pm 33.6$ & 4.03 \\
\hline$\ldots$ & HD 306787 & 4.210 & 2.84 & $5.0 \pm 0.1$ & $3.3 \pm 0.3$ & $4.09 \pm 0.09$ & $53.9 \pm 13.5$ & 4.11 \\
\hline$\ldots$ & HD 306788 & 4.303 & 3.44 & $7.3 \pm 0.1$ & $4.3 \pm 0.4$ & $4.02 \pm 0.09$ & $25.4 \pm 5.5$ & 4.01 \\
\hline$\ldots$ & HD 308743 & 4.250 & 3.10 & $5.9 \pm 0.1$ & $3.7 \pm 0.4$ & $4.06 \pm 0.09$ & $39.6 \pm 9.2$ & 4.01 \\
\hline$\cdots$ & HD 308852 & 4.192 & 3.00 & $5.2 \pm 0.1$ & $4.3 \pm 0.4$ & $3.88 \pm 0.09$ & $66.2 \pm 7.3$ & 3.83 \\
\hline$\cdots$ & NGC 3766 MG 29 & 4.260 & 3.15 & $6.1 \pm 0.1$ & $3.8 \pm 0.4$ & $4.07 \pm 0.09$ & $35.9 \pm 8.8$ & 4.11 \\
\hline$\cdots$ & NGC 3766 MG 116 & 4.255 & 2.94 & $5.5 \pm 0.2$ & $3.1 \pm 0.3$ & $4.20 \pm 0.10$ & $21.4 \pm 16.4$ & 4.22 \\
\hline$\ldots$ & NGC 3766 MG 177 & 4.311 & 3.65 & $8.1 \pm 0.2$ & $5.3 \pm 0.5$ & $3.89 \pm 0.09$ & $26.1 \pm 3.2$ & 3.60 \\
\hline
\end{tabular}

Table 6. NGC 4755: data obtained from interpolations in evolutionary diagrams given by Ekström et al. (2012) without rotation.

\begin{tabular}{lcccccccc}
\hline \hline $\begin{array}{l}\text { ID } \\
\text { Arp }\end{array}$ & $\begin{array}{c}\text { Other } \\
\text { designation }\end{array}$ & $\begin{array}{c}\log T_{\text {eff }} \\
{[\mathrm{K}]}\end{array}$ & $\log L / L_{\odot}$ & $M / M_{\odot}$ & $R / R_{\odot}$ & $\log g$ & $\begin{array}{c}\text { age } \\
{\left[10^{6} \mathrm{yr}\right]}\end{array}$ & $\log g_{\mathrm{BCD}}$ \\
\hline 1 & NSV 6008 & 4.103 & 5.05 & $18.9 \pm 0.5$ & $69.9 \pm 6.8$ & $2.02 \pm 0.10$ & $8.1 \pm 0.2$ & 2.80 \\
5 & CPD-594552 & 4.316 & 5.45 & $28.5 \pm 1.4$ & $41.4 \pm 4.0$ & $2.66 \pm 0.11$ & $5.5 \pm 0.4$ & 2.80 \\
6 & ALS 2 816 & 4.415 & 4.40 & $13.5 \pm 0.3$ & $7.8 \pm 0.7$ & $3.78 \pm 0.09$ & $10.8 \pm 1.0$ & 3.25 \\
7 & BS Cru & 4.373 & 4.47 & $13.5 \pm 0.4$ & $10.3 \pm 1.0$ & $3.54 \pm 0.10$ & $12.5 \pm 1.0$ & 3.02 \\
8 & CPD-594540 & 4.306 & 3.24 & $5.0 \pm 2.5$ & $3.4 \pm 0.3$ & $4.07 \pm 0.31$ & $13.0 \pm 10.5$ & 4.22 \\
11 & CPD-594530 & 4.194 & 2.64 & $4.5 \pm 0.1$ & $2.9 \pm 0.3$ & $4.18 \pm 0.10$ & $51.8 \pm 20.6$ & 4.30 \\
106 & BU Cru & 4.314 & 5.44 & $28.2 \pm 1.1$ & $41.6 \pm 4.0$ & $2.65 \pm 0.10$ & $5.6 \pm 0.2$ & 2.80 \\
113 & CPD-594532 & 4.413 & 3.98 & $11.0 \pm 0.5$ & $4.9 \pm 0.5$ & $4.10 \pm 0.10$ & $9.1 \pm 3.6$ & 3.89 \\
117 & CPD-594531 & 4.306 & 3.23 & $4.8 \pm 2.6$ & $3.4 \pm 0.3$ & $4.06 \pm 0.32$ & $12.2 \pm 10.3$ & 4.21 \\
201 & EI Cru & 4.375 & 4.26 & $11.9 \pm 0.3$ & $8.0 \pm 0.8$ & $3.71 \pm 0.09$ & $14.1 \pm 1.1$ & 3.26 \\
202 & CX Cru & 4.412 & 4.14 & $11.7 \pm 0.3$ & $5.9 \pm 0.6$ & $3.97 \pm 0.10$ & $11.2 \pm 2.1$ & 3.81 \\
223 & CC Cru & 4.272 & 5.34 & $25.4 \pm 0.8$ & $44.8 \pm 4.3$ & $2.54 \pm 0.10$ & $6.1 \pm 0.1$ & 2.80 \\
305 & CN Cru & 4.494 & 4.36 & $9.5 \pm 6.8$ & $5.2 \pm 0.5$ & $3.98 \pm 0.39$ & $2.3 \pm 2.1$ & 4.12 \\
306 & CPD-594559 & 4.478 & 4.77 & $18.6 \pm 0.5$ & $9.1 \pm 0.9$ & $3.79 \pm 0.10$ & $6.7 \pm 0.7$ & 3.44 \\
418 & CPD-594542 & 4.363 & 4.39 & $12.7 \pm 0.3$ & $9.9 \pm 1.0$ & $3.55 \pm 0.10$ & $13.6 \pm 0.9$ & 3.02 \\
452 & HD 312079 & 4.359 & 3.65 & $8.7 \pm 0.3$ & $4.3 \pm 0.4$ & $4.11 \pm 0.10$ & $14.4 \pm 5.4$ & 4.01 \\
454 & HD 312080 & 4.362 & 3.78 & $9.2 \pm 0.2$ & $4.9 \pm 0.5$ & $4.02 \pm 0.10$ & $17.0 \pm 3.6$ & 3.83 \\
\hline
\end{tabular}

than $3 \sigma$ of this mean value (CPD-603128, CPD-603138 and HD 100943 , which could probably be non-members), we derive a $\overline{\left(m_{\mathrm{v}}-M_{\mathrm{v}}\right)_{0}}=11.61 \pm 0.40$ and $\overline{E(B-V)}=0.22$. These parameters agree very well with most determinations found in the literature (see Table 9), and in particular with those of McSwain et al. (2008) and Schild (1970).
We constructed a HR diagram of NGC 3766 (see Fig. 5) that displays the isochrones given by Bressan et al. (1993). The age of the cluster is somewhat uncertain, but it is older than $16 \mathrm{Myr}$. Therefore, we estimated the age of the cluster by computing an average of individual stellar ages (possible members) quoted in Table 5. This estimate gives 30.7 $\pm 7.9 \mathrm{Myr}$. This age matches the 


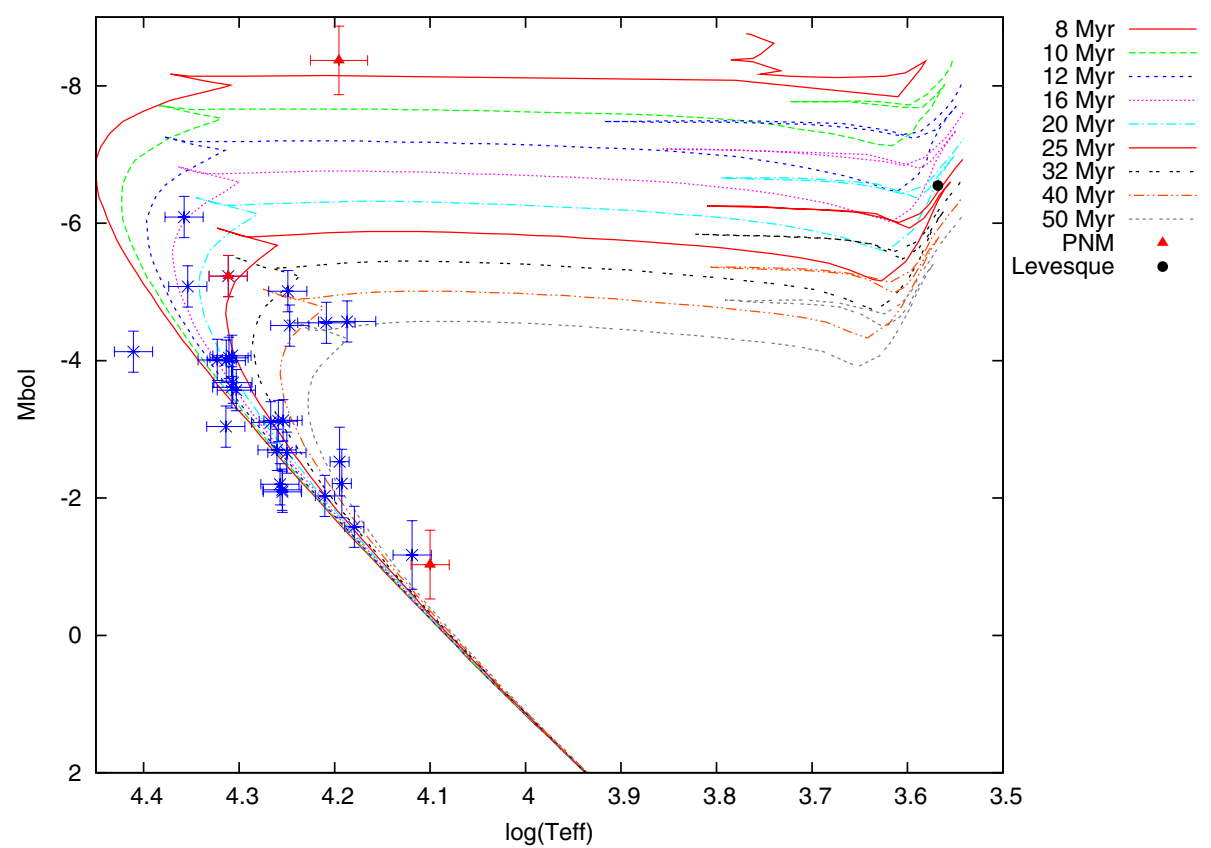

Fig. 5. HR diagram of NGC 3766. The estimated age of the cluster of $\sim 31 \mathrm{Myr}$ included the cool giant $\mathrm{CD}-603621$ reported by Levesque et al. (2005) (filled circle). The isochrone curves are given by Bressan et al. (1993). Possible members of the clusters are denoted in $\star$ (blue) symbols and possible non-members (pnm) in filled $\boldsymbol{\Lambda}$ (red).

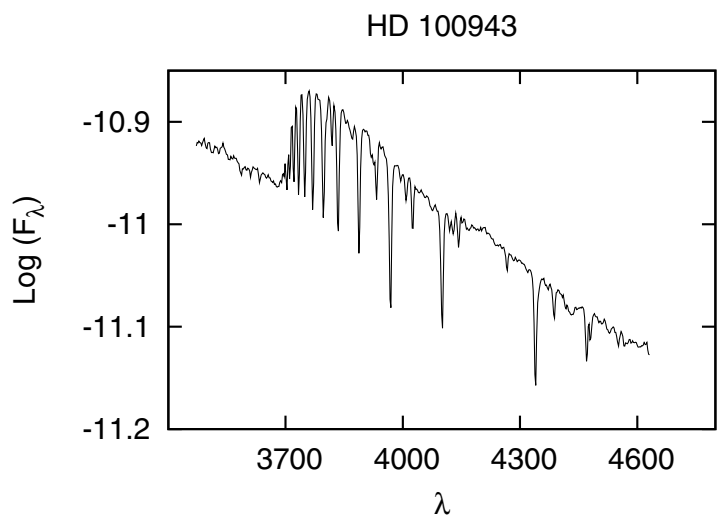

ALS 2401

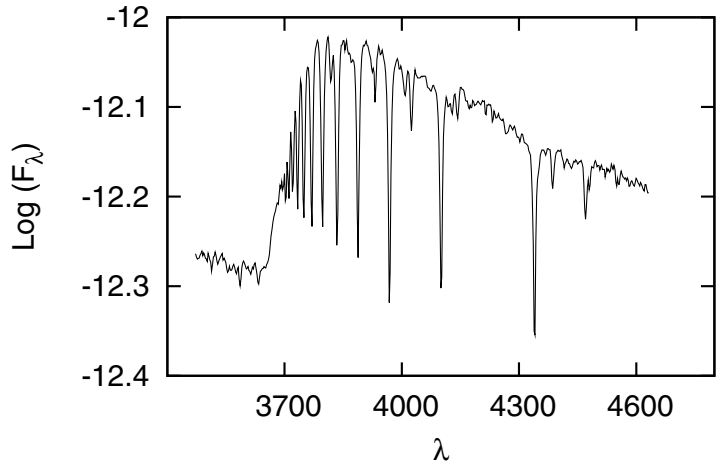

HD 306657

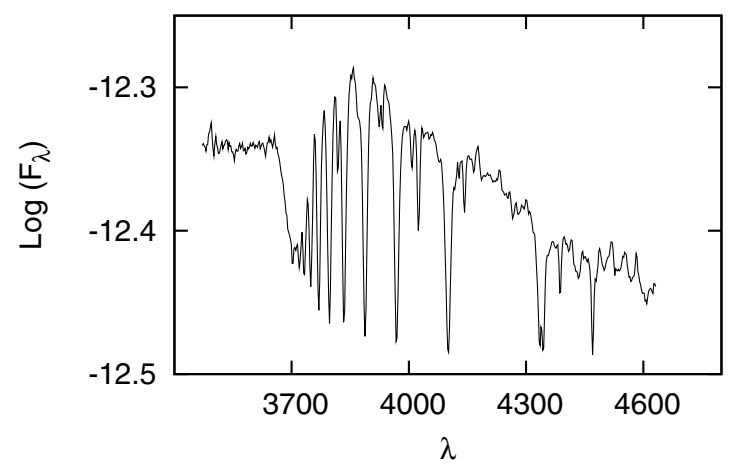

HD 308852

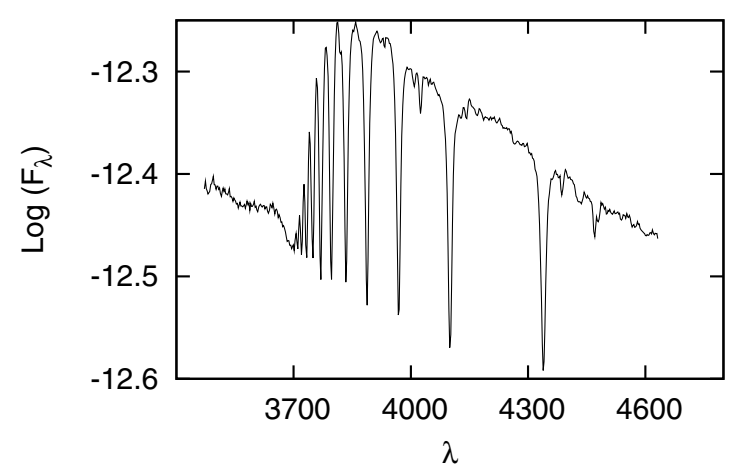

Fig. 6. NGC 3766: stars showing a second component of the Balmer discontinuity.

location of the red supergiant CD-603621 reported by Levesque et al. (2005) (filled circle symbol).

Finally, from this spectroscopic study we found that HD 100943 and ALS 2401 show the second Balmer discontinuity in absorption, while HD 306657 and HD 308852 are seen in emission, indicating stars with the Be phenomenon (see Fig. 6). HD 100943 is a known B supergiant star but HD 308852 has not previously been reported as a Be star.

\subsubsection{NGC 4755}

The open cluster NGC $4755\left(\alpha=12^{\mathrm{h}} 53^{\mathrm{m}} 42^{\mathrm{s}}\right.$ and $\left.\delta=-60^{\circ} 22^{\prime}\right)$, also known as the $\kappa$ Crucis cluster or "Jewel Box" is a highly concentrated group of stars with a very considerable difference in luminosity. Photometric and/or spectroscopic studies were performed by Arp \& van Sant (1958), Hernández (1960), Feast (1963), Graham (1967), Schild (1970), Shobbrook (1984), 

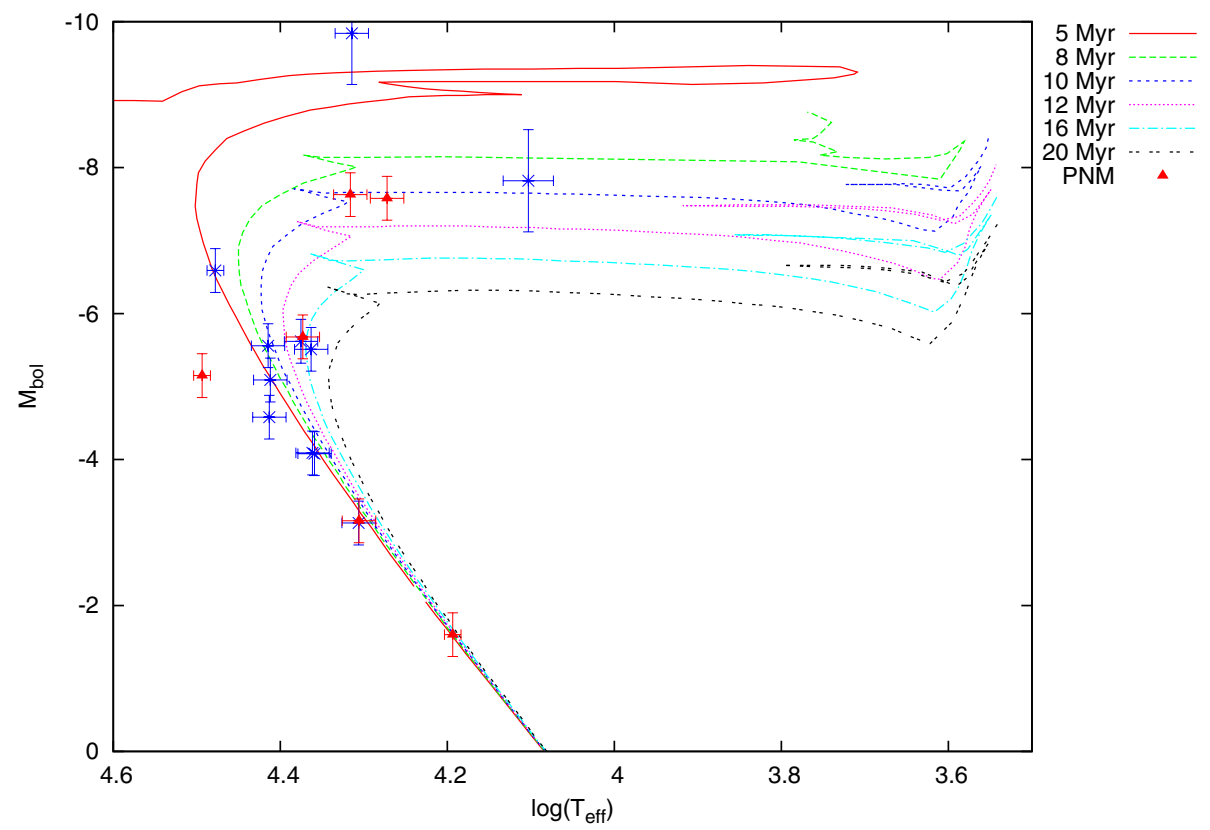

Fig. 7. HR diagram of NGC 4755 . The estimated age of the cluster is $\sim 11$ Myr. The isochrone curves are given by Bressan et al. (1993). Possible members of the clusters are denoted in $\star$ (blue) symbols and possible non-members (pnm) in filled $\boldsymbol{\Lambda}$ (red).

Table 9. Summary: determinations of color excess, distance, and age of NGC 3766.

\begin{tabular}{lcccc}
\hline \hline Works & $\begin{array}{c}E(B-V) \\
{[\mathrm{mag}]}\end{array}$ & $\begin{array}{c}\left(m_{\mathrm{v}}-M_{\mathrm{V}}\right)_{0} \\
{[\mathrm{mag}]}\end{array}$ & $\begin{array}{c}\text { Distance } \\
{[\mathrm{pc}]}\end{array}$ & $\begin{array}{c}\text { Age } \\
{[\mathrm{Myr}]}\end{array}$ \\
\hline Sher (1962) & $0.19 \pm 0.01$ & 11.4 & 1900 & 10 \\
Sher (1965) & $0.19 \pm 0.005$ & 11.4 & 1900 & $\ldots$ \\
Schild (1970) & 0.22 & $11.6 \pm 0.2$ & 2200 & $\ldots$ \\
Winnenburg (1973) & 0.16 & 10.92 & 1530 & $\ldots$ \\
Yilmaz (1976) & $\ldots$ & 12.25 & 2150 & 22 \\
Shobbrook (1985) & 0.20 & $11.4 \pm 0.1$ & $1900 \pm 100$ & $\ldots$ \\
Moitinho et al. (1997) & $0.20 \pm 0.10$ & $11.73 \pm 0.33$ & $2218 \pm 335$ & 24 \\
McSwain et al. (2008) & $0.223 \pm 0.03$ & $11.6 \pm 0.2$ & $2100 \pm 200$ & $\ldots$ \\
This work & 0.22 & $11.61 \pm 0.40$ & $2100 \pm 390$ & 31 \\
\hline
\end{tabular}

Sanner et al. (2001), Evans et al. (2005), and Dufton et al. (2006).

A first estimate of the distance modulus considering 17 stars is $\left(m_{\mathrm{v}}-M_{\mathrm{v}}\right)_{0}=12.15 \pm 0.39 \mathrm{mag}$ and $\overline{E(B-V)}=0.30$. By rejecting the pnm stars CPD-594552, BS Cru, CPD-594540, CPD-594530, CC Cru, and CN Cru, which are more than $3 \sigma \simeq$ 1.17 mag of the mean distance modulus, that is $\left(m_{\mathrm{v}}-M_{\mathrm{v}}\right)_{0}<$ $11 \mathrm{mag}$ or $\left(m_{\mathrm{v}}-M_{\mathrm{v}}\right)_{0},>13.3 \mathrm{mag}$, we obtain $\overline{\left(m_{\mathrm{v}}-M_{\mathrm{v}}\right)_{0}}=$ $12.10 \pm 0.39 \mathrm{mag}$ and $\overline{E(B-V)}=0.30$.

In Fig. 7 we show the isochrones that better fit the NGC 4755 HR diagram and derive ages between 5 and 16 Myr. A better estimate of $11.2 \pm 2.7$ Myr can be obtained averaging individual stellar ages of possible members (see Table 6).

The inferred distance modulus and age found in this work for this cluster agree with previous estimates (see Table 10).

The spectral types derived from the BCD method agree very well with the determinations by Evans et al. (2005) using the MK system, with the $\mathrm{H} \gamma$ equivalent widths and the Balona \& Crampton (1974) calibration, for 14 stars in common. The exception is CPD-594530, for which we determine a spectral type of B6 V in accordance with Feast (1963) (B8 V).

Spectra with a second Balmer discontinuity are observed in NSV 6008 and CPD-594559, which are a B7 Ia supergigant and a known Be star (Feast 1963), respectively (see Fig. 8).
Table 10. Summary: determinations of color excess, distance, and age of NGC 4755 .

\begin{tabular}{lcccc}
\hline \hline Works & $\begin{array}{c}E(B-V) \\
{[\mathrm{mag}]}\end{array}$ & $\begin{array}{c}\left(m_{\mathrm{v}}-M_{\mathrm{v}}\right)_{0} \\
{[\mathrm{mag}]}\end{array}$ & $\begin{array}{c}\text { Distance } \\
{[\mathrm{pc}]}\end{array}$ & $\begin{array}{c}\text { Age } \\
{[\mathrm{Myr}]}\end{array}$ \\
\hline Arp \& van Sant (1958) & $0.31 \pm 0.3$ & $9.6 \pm 0.3$ & 830 & 3 \\
Hernández (1959) & $\ldots$ & 12.1 & $\ldots$ & $\ldots$ \\
Wallenquist (1959) & $\ldots$ & $10.8 \pm 0.3$ & $\ldots$ & $\ldots$ \\
Hernández (1960) & 0.44 & 11.6 & $\ldots$ & $\ldots$ \\
Becker (1961) & 0.00 & 11.0 & $\ldots$ & $\ldots$ \\
Becker (1963) & 0.31 & 10.1 & $\ldots$ & $\ldots$ \\
Buscombe (1963) & 0.40 & 11.8 & $\ldots$ & 6 \\
Feast (1963) & $0.48 \pm 0.01$ & $11.86 \pm 0.14$ & 2360 & $\ldots$ \\
Kennedy (1966) & 0.46 & 11.9 & $\ldots$ & $\ldots$ \\
Barbaro et al. (1967) & $\ldots$ & $\ldots$ & $\ldots$ & 8 \\
Graham (1967) & 0.40 & $11.76 \pm 1.0$ & $\ldots$ & $\ldots$ \\
Schild (1970) & 0.42 & 11.6 & $\ldots$ & $\ldots$ \\
Becker \& Fenkart (1971) & 0.31 & 10.1 & $\ldots$ & $\ldots$ \\
Perry et al. (1976) & $0.40 \pm 0.01$ & $11.8 \pm 0.2$ & $\ldots$ & 7 \\
Shobbrook (1984) & 0.38 & $11.4 \pm 0.1$ & $\ldots$ & $\ldots$ \\
Sanner et al. (2001) & $0.36 \pm 0.02$ & $11.6 \pm 0.2$ & $2100 \pm 200$ & $10 \pm 5$ \\
McSwain \& Gies (2005) & 0.38 & 11.80 & 2290 & 7 \\
This work & 0.30 & $12.10 \pm 0.39$ & $2630 \pm 475$ & 11 \\
\hline
\end{tabular}

\section{Discussion}

\subsection{NGC 3766}

Our estimates of the distance and age for the cluster agree very well with previous determinations. However, fundamental parameter values of individual stars are fairly scarce in the literature. The comparison of individual BCD $T_{\text {eff }}$ and $\log g$ values with those derived by McSwain et al. (2008) for 14 stars in common presents agreements in seven stars with discrepancies lower than $1500 \mathrm{~K}$ or 0.25 dex, respectively (see Table 11).

Among the stars we considered pnm, the supergiant HD 100943 was also reported as a background star by Schild (1970).

We found that individual $\mathrm{BCD} M_{\mathrm{v}}$ measurements agree well with those that we derived from the Balona \& Crampton (1974) calibration, which were obtained using our BCD spectral type classifications (see Fig. 9 and Table 12). In addition, our $\left(m_{\mathrm{v}}-M_{\mathrm{v}}\right)_{0}$ estimates for the individual stars agree with the values given by Schild (1970) and Shobbrook (1985) (see Table 13). 

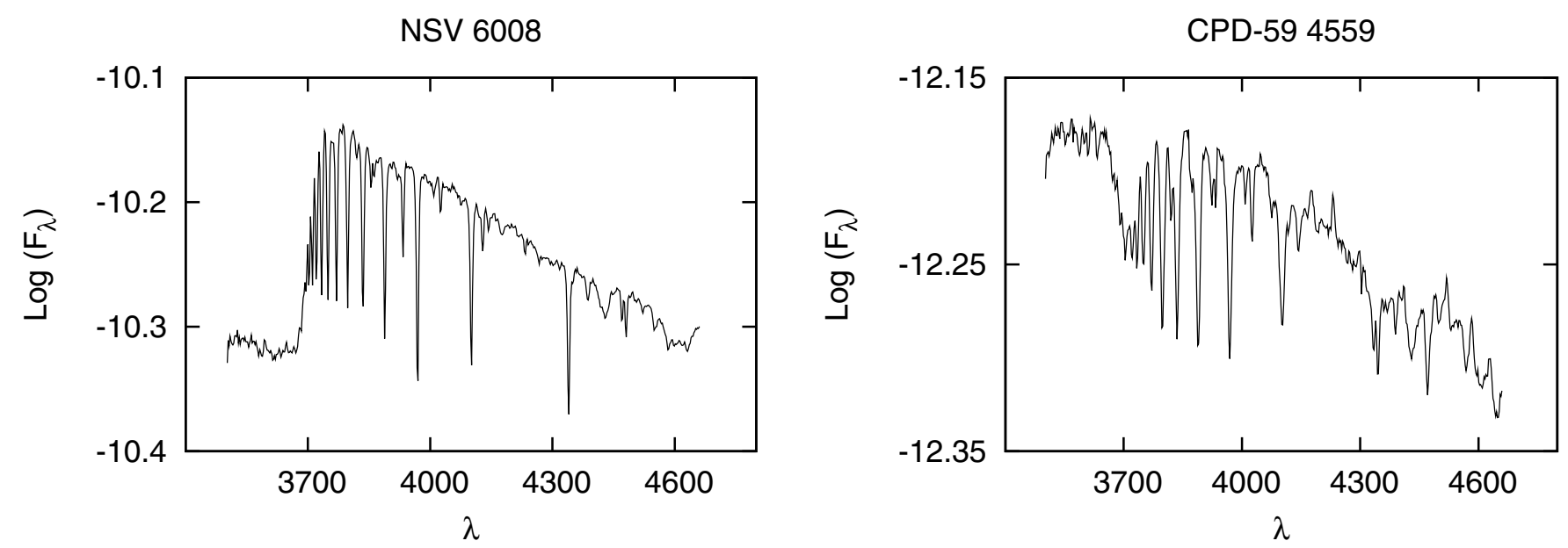

Fig. 8. NGC 4755: stars showing a second component of the Balmer discontinuity.

Table 11. NGC 3766: comparison between BCD $T_{\text {eff }}$ and $\log g$ values and measurements obtained in the literature.

\begin{tabular}{lccc|cc}
\hline \hline ID & Other & \multicolumn{2}{c|}{ This work } & \multicolumn{2}{c}{ McSwain et al. (2008) } \\
Ahmed & designation & $T_{\text {eff }}$ & $\log g$ & $T_{\text {eff }}$ & $\log g$ \\
\hline 1 & ALS 2 398 (pe) & 16170 & 2.81 & 18725 & 3.34 \\
5 & ALS 2 408 (v) & 17660 & 2.80 & 18399 & 3.30 \\
24 & CPD-603131 & 15110 & 4.24 & 16760 & 3.98 \\
26 & CPD-603125(ne) & 21040 & 3.81 & 18274 & 3.49 \\
27 & CPD-603128 & 20580 & 3.49 & 18817 & 3.31 \\
30 & CPD-603135 & 18080 & 4.11 & 11714 & 4.33 \\
151 & CD-603626 & 17950 & 4.01 & 17834 & 3.82 \\
169 & HD 100 969 & 20480 & 2.80 & 19420 & 3.48 \\
239 & HD 306 798 (Be) & 20300 & 3.62 & 19660 & 3.73 \\
240 & ALS 2 401*(Be) & 15380 & 2.80 & 16301 & 3.51 \\
264 & HD 306 657*(Be) & 25740 & 4.04 & 19580 & 4.00 \\
291 & HD 306 793 (e) & 18490 & 4.04 & 18995 & 4.02 \\
317 & HD 306785 & 20580 & 3.62 & 17071 & 3.10 \\
$\ldots$ & NGC 3766 MG 29 & 18210 & 4.11 & 20140 & 4.66 \\
\hline
\end{tabular}

Notes. (Be) Be star. (e) Emission line star. (v) Eruptive variable. (pe) Sharp $\mathrm{H} \gamma$ line and incipient emission at $\mathrm{H} \beta$. (ne) Moderate emission at $\mathrm{H} \beta .{ }^{(*)}$ Stars with double Balmer discontinuity.

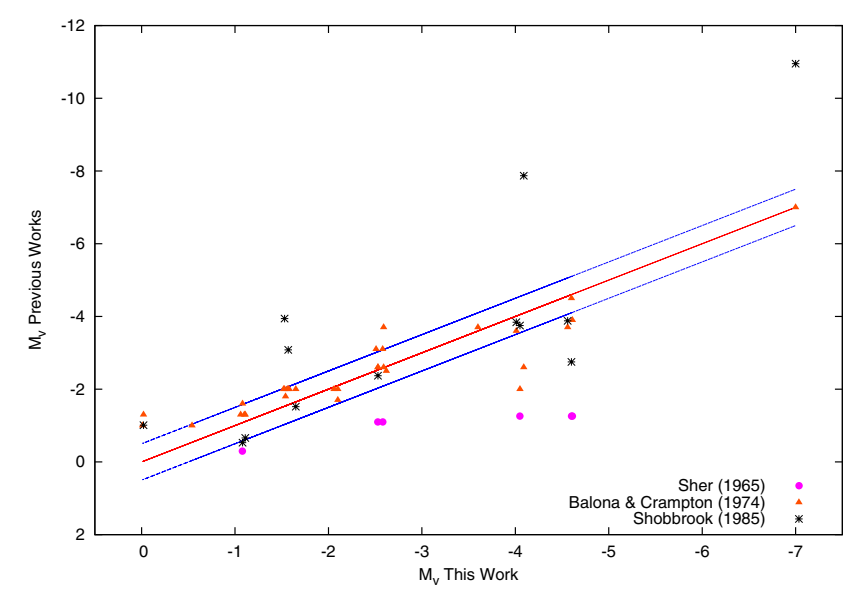

Fig. 9. NGC 3766: correlation among different $M_{\mathrm{v}}$ estimates.

However, large discrepancies are obtained with the measurements by Sher (1965).

The mean BCD color excess derived via the color gradient excess also agrees with $i)$ the value $\overline{(B-V)-(B-V)_{0}}=$ 0.21 , where $(B-V)$ are the observed color index taken

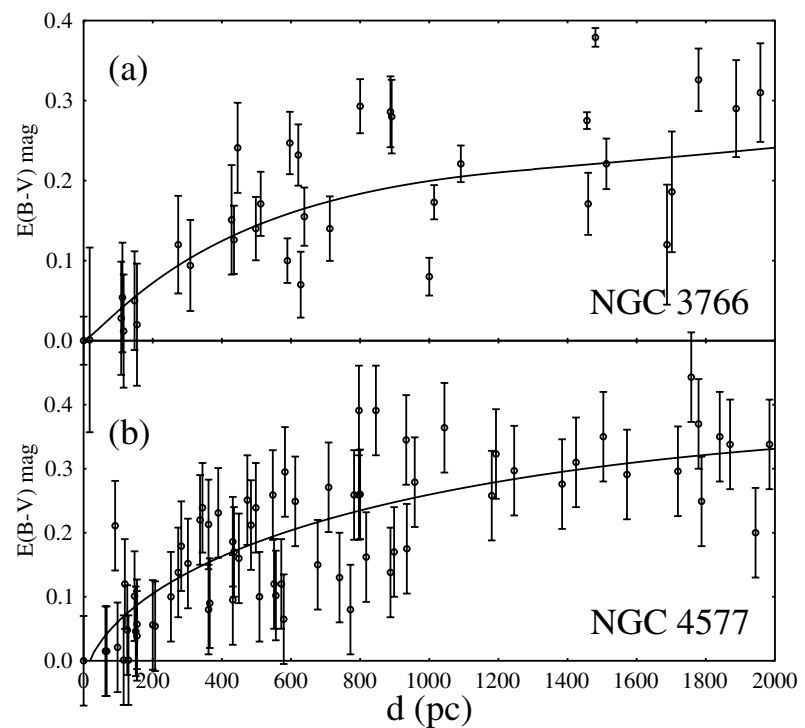

Fig. 10. Relation between the ISM color excess $E(B-V)_{\mathrm{ISM}}$ and the distance.

from the SIMBAD database and $(B-V)_{0}$ were obtained using our BCD temperature data and the Flower (1996) colortemperature calibration and $i i)$ color excess, $E(B-V)_{\text {ISM }}$, derived from the interstellar absorption curve as a function of the distance (Neckel et al. 1980) in the direction of the studied clusters (see Fig. 10). These curves were obtained with neighboring stars in a cone of $0.5^{\circ}$ in the direction of the two clusters using intrinsic color $-(B-V)$ and absolute magnitude $-M_{\mathrm{V}}$ calibrations given by Deutschman et al. (1976). The color excess and the distance were computed by an iterative procedure in Pogson's law. For NGC 3766, we obtained an $E(B-V)_{\text {ISM }}=0.251$ and cluster distance of $2204 \mathrm{pc}$.

\subsection{NGC 4755}

The discrepancies found among the distance moduli obtained by different authors are strongly related to the reddening measurements, since the color excess has the contribution of two components: one due to the interstellar material and other due to the circumstellar envelope of Be stellar population. Whilst photometric methods use the mean reddening values as a pure interstellar reddening, the BCD spectroscopic system enables us 
Table 15. NGC 4755: comparison of our $T_{\text {eff }}$ and $\log g$ determinations with data from literature.

\begin{tabular}{lccc|cc}
\hline \hline ID & Other & \multicolumn{2}{c|}{ This work } & \multicolumn{2}{c}{ Dufton et al. (2006) } \\
Arp & designation & $T_{\text {eff }}$ & $\log g$ & $T_{\text {eff }}$ & $\log g$ \\
\hline 1 & NSV 6008* (vs) & 12680 & $<2.80$ & 13500 & 2.00 \\
5 & CPD-594552 & 20710 & $<2.80$ & 21500 & 3.15 \\
6 & ALS 2 816 (vc) & 25980 & 3.25 & 25000 & 3.90 \\
7 & BS Cru (vc) & 23610 & 3.02 & 25300 & 4.00 \\
8 & CPD-594540 & 20220 & 4.22 & 22500 & 4.10 \\
11 & CPD-594530 & 15620 & $>4.30$ & 15200 & 3.85 \\
106 & BU Cru & 20620 & $<2.80$ & 20000 & 2.75 \\
113 & CPD-594532(vc) & 25890 & 3.89 & $\ldots$ & $\ldots$ \\
117 & CPD-594531 & 20240 & 4.21 & 18800 & 3.70 \\
201 & EI Cru (vc) & 23730 & 3.26 & 25000 & 4.10 \\
202 & CX Cru (vc) & 25820 & 3.81 & $\ldots$ & $\ldots$ \\
223 & CC Cru (ve) & 18710 & $<2.80$ & 16900 & 2.80 \\
305 & CN Cru (eb) & 31170 & 4.12 & 25000 & 3.70 \\
306 & CPD-59 559* (Be) & 30040 & 3.44 & $\ldots$ & $\ldots$ \\
418 & CPD-594542(vs) & 23070 & 3.02 & 22500 & 3.90 \\
452 & HD 312 079 & 22860 & 4.01 & 22500 & 4.10 \\
454 & HD 312 080 & 22990 & 3.83 & 19500 & 3.80 \\
\hline
\end{tabular}

Notes. (Be) Be star. (vs) Variable star. (vc) Variable star of beta Cep type. (ve) Ellipsoidal variable star. (eb) Eclipsing binary of beta Lyr type. ${ }^{(*)}$ Stars with double Balmer discontinuity.

to determine individual measurements corrected for both circumstellar absorption/emission and interstellar extinction. The mean BCD color excess derived via the color gradient excess is slightly lower than $\overline{(B-V)-(B-V)_{0}}=0.39$, which was obtained with the observed $(B-V)$ values taken from Dachs \& Kaiser (1984) and the $(B-V)_{0}$ estimates derived using our BCD temperature data and the Flower (1996) colortemperature calibration. Although this last estimate corresponds to measurements obtained in quite different epochs! The derived color excess $E(B-V)_{\mathrm{ISM}}=0.33$ using the neighboring-star method is also close to that obtained in Sect. 4.2.2, as well as the distance, which in this case is $d=2460 \mathrm{pc}$.

The spectral types derived from the BCD method and those obtained by Evans et al. (2005) using the MK system agree excellently. However, $T_{\text {eff }}$ values obtained with the BCD method show in general a good agreement with the values derived by Dufton et al. (2006), although some discrepancies of about of $2000 \mathrm{~K}$ are observed in subtypes B1 and B2; i.e. NGC 4755223 and NGC 4755454 (see Table 15). Note that the BCD method becomes less sensitive for the hottest stars in our sample and thus the error margin increases. The discrepancies in $\log g$ shown in the Table 15 are lower than $\sim 0.5$, except for the star NGC 4755201.

For the individual absolute magnitude estimates we found a good agreement for stars with luminosity classes between III and V. Discrepancies larger than 0.5 are mainly found in luminosity classes I and II (see Table 16 and Fig. 11).

\subsection{The bolometric magnitudes and effective gravities}

The bolometric magnitude provides a global way to test the BCD calibrations of fundamental parameters. To this end we calculated the absolute bolometric magnitude of stars, called here $M_{\text {bol }}(\langle d\rangle)$, using the distances $\langle d\rangle$ adopted in this paper of the program open clusters, the individual stellar apparent visual magnitude $m_{\mathrm{V}}$, its color excess $E(B-V)$, and the respective BCD effective temperature $T_{\text {eff }}\left(\lambda_{1}, D\right)$ to estimate the corresponding bolometric corrections $B C\left(T_{\text {eff }}\right)$. Figure 12 shows $M_{\text {bol }}(\langle d\rangle)$ as a

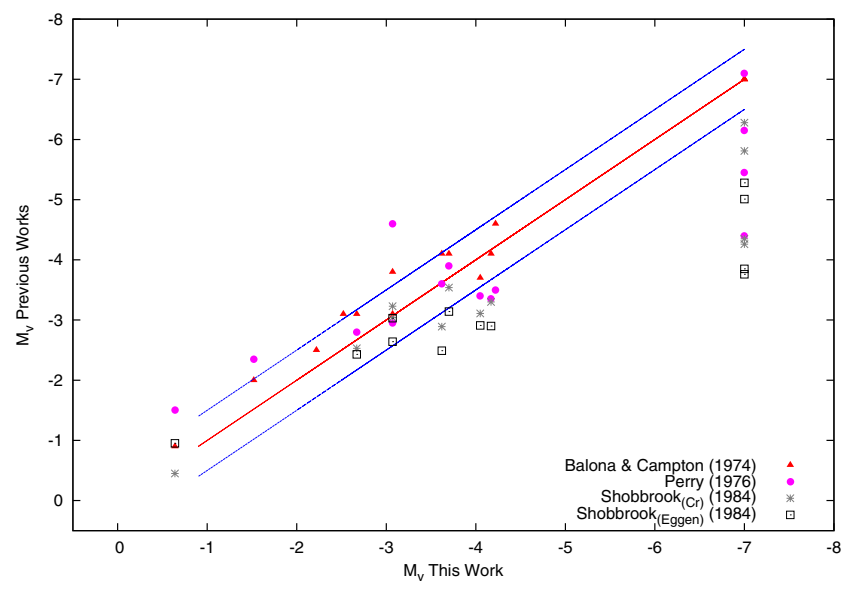

Fig. 11. NGC 4755: correlation among different $M_{\mathrm{v}}$ estimates.

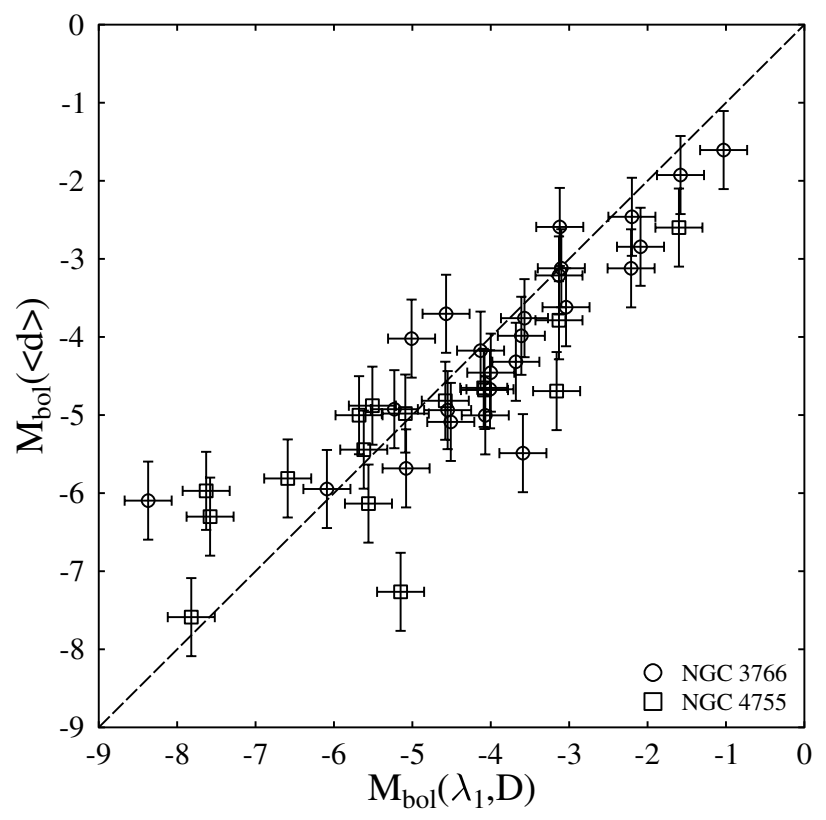

Fig. 12. Calculated $M_{\text {bol }}(\langle d\rangle)$ absolute bolometric magnitudes against $M_{\text {bol }}\left(\lambda_{1}, D\right)$ of stars in both studied open clusters. Circles are for stars in NGC 3766, while squares are for stars in NGC 4755.

function of $M_{\mathrm{bol}}\left(\lambda_{1}, D\right)$, where the uncertainties of the calculated magnitudes are also given. Despite the scatter of points, which on average amounts to roughly the value of the individual uncertainties, we see no strong systematic deviation over the whole domain of magnitudes, which reveal a certain degree of internal consistency of the adopted $M_{\mathrm{bol}}\left(\lambda_{1}, D\right)$ calibration.

The bolometric magnitude $M_{\text {bol }}\left(\lambda_{1}, D\right)$ and $T_{\text {eff }}\left(\lambda_{1}, D\right)$ (whose calibration was extensively discussed in Zorec et al. 2009) were used as input parameters to the evolutionary tracks for non-rotating stars calculated by Schaller et al. (1992). We thus estimated the stellar radii $R / R_{\odot}$ and masses $M / M_{\odot}$, from which we could estimate the $\log g_{\text {evol }}$ of each star. The stellar surface gravity is another fundamental parameter that enabled us to test the current $\log g\left(\lambda_{1}, D\right)$ calibration. The comparison of $\log g_{\text {evol }}$ with $\log g\left(\lambda_{1}, D\right)$ is shown in Fig. 13. Contrary to the test of bolometric magnitudes, we see in Fig. 13 that there is a systematic deviation between the two surface gravity estimates in the sense that $\log g_{\text {evol }}>\log g\left(\lambda_{1}, D\right)$ in the $2.7 \lesssim \log g \lesssim 4.50$ dex interval with differences that steadily 


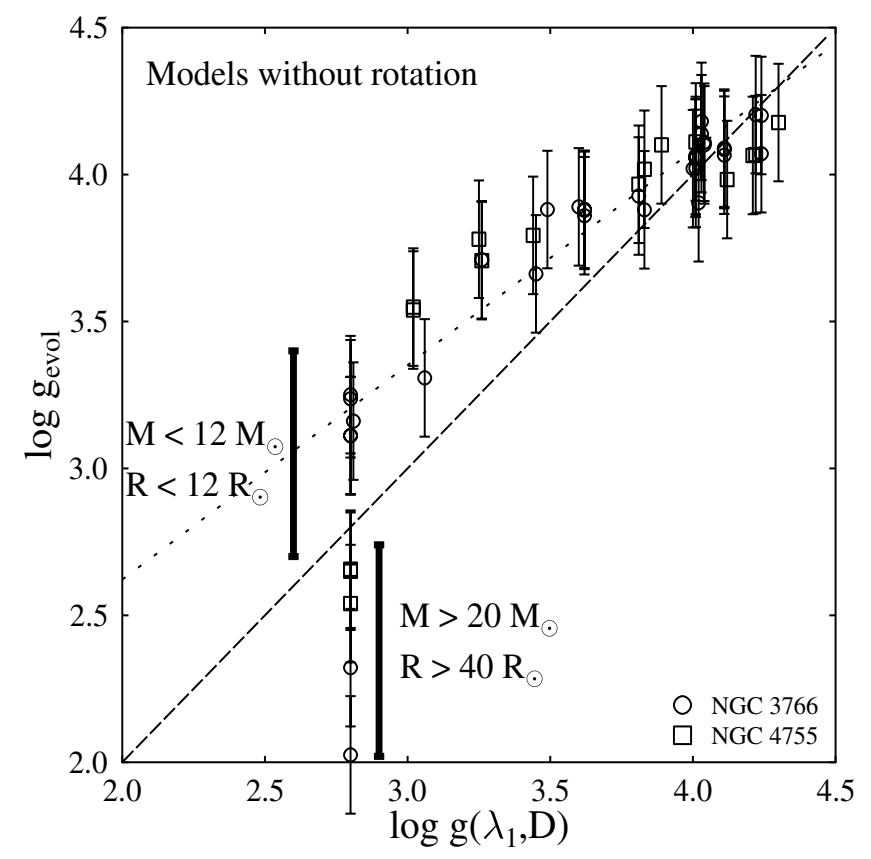

Fig. 13. Estimated $\log g_{\text {evol }}$ parameters with models of stellar evolution against $\log g\left(\lambda_{1}, D\right)$ obtained with the BCD calibrations. As in Fig. 12, circles denote stars in NGC 3766, while squares denote stars in NGC 4755. We also indicate the marked stellar masses and radii for which $\log g_{\text {evol }}>\log g\left(\lambda_{1}, D\right)$ and those where $\log g_{\text {evol }}<\log g\left(\lambda_{1}, D\right)$. The evolutionary models used to infer stellar masses are those by Ekström et al. (2012) without rotation.

grow as $\log g$ ranges from 4.5 to 2.7 dex. However, we see that at $\log g\left(\lambda_{1}, D\right) \simeq 2.75$ there are two different deviations: stars with masses $M \lesssim 12 M_{\odot}$ and radii $R \lesssim 12 R_{\odot}$ have $\log g_{\text {evol }}>\log g\left(\lambda_{1}, D\right)$, while those with $M \gtrsim 20 M_{\odot}$ and radii $R \gtrsim 40 R_{\odot}$ have $\log g_{\text {evol }}<\log g\left(\lambda_{1}, D\right)$. We note that the $\log g\left(\lambda_{1}, D\right)$ calibration was established using $\log g_{\text {atm }}$ parameters obtained by fitting models of stellar atmospheres to spectral lines, mainly Balmer lines. A similar systematic deviation between $\log g_{\text {evol }}$ and $\log g_{\text {atm }}$ has already been noted also for A-type stars by Gerbaldi \& Zorec (1993). The comparison displayed in Fig. 13 shows that there must be a difference in the interpretation of the $\log g$ parameters if they are calculated with stellar atmosphere models or with models of stellar structure, because the $\log g_{\text {evol }}$ quantities would relate on average to deeper stellar layers than $\log g_{\text {atm }}$, for stars with $M \lesssim 12 M_{\odot}$ and radii $R \lesssim 12 R_{\odot}$. A reverse situation would occur for evolved massive stars with $M \gtrsim 20 M_{\odot}$ and $R \gtrsim 40 R_{\odot}$.

It is worth noting that rotation, which we neglected in the present approach, would act with systematic differences that range in the opposite way as noted above for stars with $M \lesssim$ $12 M_{\odot}$. The apparent stellar parameters of rotating stars derived using models without rotation produce higher apparent bolometric luminosities and lower effective temperatures than found for the non-rotating stellar counterparts (Frémat et al. 2005; Zorec et al. 2005), which results on average lower $\log g_{\text {evol }}$ determinations than for the non-rotating counterparts. The same diagram obtained with masses derived from evolutionary tracks and the same apparent stellar fundamental parameters produces extremely marginal differences.

The noted differences in the estimate of the $\log g_{\text {evol }}$ and $\log g\left(\lambda_{1}, D\right)$ (or $\log g_{\text {atm }}$ ) parameters will be discussed in detail a separate paper.
Table 19. Stellar masses and time spent in the main sequence inferred from different evolutionary models.

\begin{tabular}{l|cc|cc}
\hline \hline$\left(M / M_{\odot}\right)_{\Omega=0}$ & \multicolumn{2}{|c|}{$\left(M / M_{\odot}\right)_{\mathrm{B}}$} & \multicolumn{2}{|c}{$t_{\mathrm{MS}}(\mathrm{yr})$} \\
& $t / t_{\mathrm{MS}}=1 / 4$ & $t / t_{\mathrm{MS}}=0.8$ & $\Omega=0$ & $\mathrm{~B}$ \\
\hline 3.0 & 3.3 & 3.2 & $3.16 \times 10^{8}$ & $4.55 \times 10^{8}$ \\
5.0 & 5.3 & 5.1 & $8.62 \times 10^{7}$ & $1.23 \times 10^{8}$ \\
7.0 & 7.6 & 7.4 & $4.14 \times 10^{7}$ & $5.67 \times 10^{7}$ \\
9.0 & 9.8 & 9.5 & $2.61 \times 10^{7}$ & $3.43 \times 10^{7}$ \\
15.0 & 16.4 & 15.7 & $1.09 \times 10^{7}$ & $1.44 \times 10^{7}$ \\
20.0 & 21.4 & 20.7 & $7.70 \times 10^{6}$ & $9.95 \times 10^{6}$ \\
\hline
\end{tabular}

Notes. $\Omega=0$ indicates models by Ekström et al. (2012) without rotation. B indicates models by Bertelli et al. (2009). Age fractions $t / t_{\mathrm{MS}}$ refer to models by Ekström et al. (2012).

\subsection{The stellar masses and ages}

To derive stellar masses and ages we used the evolutionary tracks calculated by Ekström et al. (2012), since these authors provided uniform models for masses ranging from 1 to $120 M_{\odot}$ that avoid using extrapolated values. We might wonder, however, what the stellar parameters would have been if we had used other calculations of stellar evolution, which might have different physical inputs. In Table 19 we compare values of stellar masses inferred from Ekström et al.'s calculations, for metallicity $Z=0.014$, and from Bertelli et al.'s (2009) models, calculated for $Z=0.017$. The masses are obtained for two reference evolutionary phases $t / t_{\mathrm{MS}}=0.25$ and 0.8 (where $t / t_{\mathrm{MS}}$ is the fraction of time that every star has lived in the main sequence and $t_{\mathrm{MS}}$ is the time spent by the star in the main sequence phase). The quoted initial model metallicities are currently assumed solar ones. Figure 14 shows the corresponding evolutionary tracks computed with and without rotation by Ekström et al. (2012) (full and dashed lines, respectively) and the Bertelli et al. models (in dotted lines). We see that systematic deviations exist in the sense that the Bertelli et al. models produce slightly larger stellar masses. These authors only provided models for masses ranging form 0.15 to $20 M_{\odot}$ and 39 chemical compositions. Differences in the estimate of masses and ages derived with models with rotation can be seen in Tables 7 and 8 .

It is also interesting to consider the ages that would be inferred with each model. Table 19 gives the time $t_{\mathrm{MS}}$ that a star can spend in the main sequence. Curiously, although the masses obtained by interpolation using Bertelli et al.'s models are larger, their ages are also longer, from $30 \%$ to $20 \%$ as masses range from 3 to $20 M_{\odot}$, respectively.

Finally, we emphasize again that the apparent fundamental parameters, i.e. those derived from observations as the stars were not rotating, can depend more or less strongly on the effects induced by the rotation through the stellar geometrical deformation, gravitational darkening, internal evolution pace, surface chemical abundance, etc. In such a case each observed fundamental parameter needs to be treated properly to infer the actual stellar mass, age and size. All they require is to deal with models of stellar evolution with rotation calculated for the appropriate $\Omega / \Omega_{\mathrm{c}}$ ratio at the ZAMS that at the current stellar evolutionary phase reproduces the observed $V \sin i$ parameter, for which we also need to iterate the inclination angle $i$. To carry out these procedure, at least three independent parameters have to be determined from observations: the apparent effective temperature $T_{\text {eff }}(i)$, the apparent effective surface gravity $\log g_{\text {eff }}(i)$, the apparent bolometric luminosity $\log L(i) / L_{\odot}$, or the apparent $V \sin i$. Since for most cases $\log L(i) / L_{\odot}$ cannot be easily 


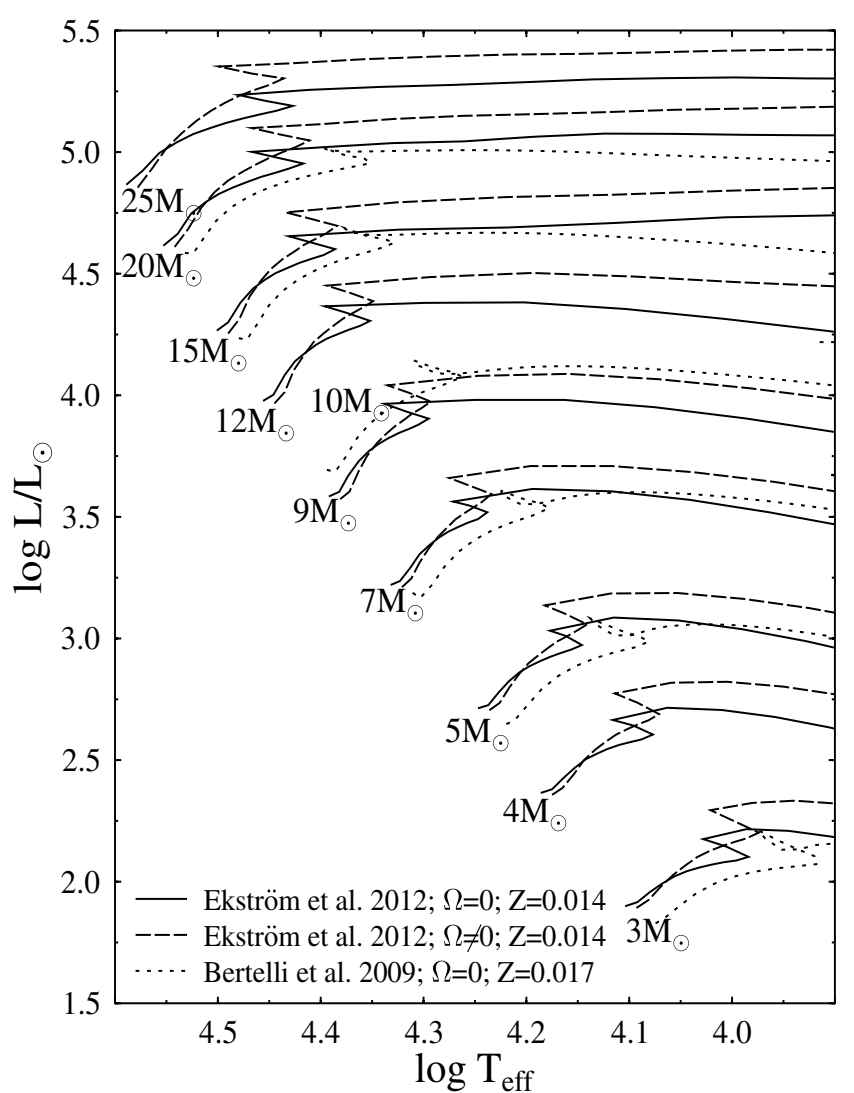

Fig. 14. Comparison of evolutionary tracks calculated by Ekström et al. (2012), with and witout rotation, and those of Bertelli et al. (2009). Systematic differences could appear in the determination of stellar masses and ages when one or the other series of evolutionary models are used. They are not significant compared to those that can still arise from rotational effects once the apparent fundamental parameters are consistently treated for the rotational effects.

estimated, the operation can be carried out assuming $\Omega / \Omega_{\mathrm{c}}$ ratios for the present-day stellar rotation. This requires a long and specific iteration procedure that is beyond the purposes of the present work (Frémat et al. 2005; Zorec et al. 2005).

\subsection{The second Balmer discontinuity}

The spectroscopic study of Be stars in open clusters is of great relevance since it will provide important keys on their formation and evolutionary stage through the particular conditions they originated from: similar age and chemical composition. On the other hand, the effects of other stellar properties could also be more easily determined than for isolated stars. Concerning these properties, it is worth mentioning some peculiarities related to the behavior of the second Balmer discontinuity. Although there is no formal statistical study, from the work of Moujtahid et al. (1998) we can deduce the following trend in isolated Be stars: taking into account the presence of the second discontinuity (for instance, cases with $|\delta D|>0.05$ ), we found that the second Balmer discontinuity is in emission when $V \sin i<200 \mathrm{~km} \mathrm{~s}^{-1}$ and in absorption when $V \sin i>200 \mathrm{~km} \mathrm{~s}^{-1}$. However, exceptions to this trend are observed in some Be stars of NGC 3766 and NGC 4755. CPD-594559 shows the second Balmer discontinuity in emission and has a high projected rotation velocity and the two supergiants (HD 100943 and NSV 6008) exhibit the second discontinuity in absorption. Another exception to this rule was reported in the literature by Divan et al. (1983) for HD 60848, an O-type star with $\delta D<0$ (emission) and $V \sin i=360 \mathrm{~km} \mathrm{~s}^{-1}$. These peculiar cases could provide information on the physical and geometrical properties of the circumstellar environment that produced the second discontinuity.

\section{Conclusions}

We used the BCD system, which is based on measurable quantities in the stellar continuum spectrum around the Balmer's discontinuity and enables one to determine the fundamental parameters of the stars in a direct way. To this end, we carried out a complete study of the members of two known young open clusters (NGC 3766 and NGC 4755), deriving not only the fundamental parameters of the stars but the cluster's distance modulus and age as well. For some of these stars $T_{\text {eff }}, \log g$, spectral types, $E(B-V), M_{\mathrm{v}}, M_{\mathrm{bol}}$, ages, masses, stellar radii, and stellar rotation have been obtained for the first time (Tables 3-6).

Detailed results on the individual star population will enable us to improve, in a near future, our knowledge on the formation and evolution of the clusters. Future statistical studies of the star members will provide evidence on the initial mass, luminosity, and angular momentum distributions of open clusters.

The cluster parameters obtained via the BCD method agree very well with the parameters derived from classical methods based on photometric data. The main results for the cluster parameters are summarized below:

- For NGC 3766 we obtained $\overline{\left(m_{\mathrm{v}}-M_{\mathrm{v}}\right)_{0}}=11.61 \pm 0.40$, a mean value of $\overline{E(B-V)}=0.22$ and an age of $30.7 \mathrm{Myr}$.

- For NGC 4755 we obtained $\overline{\left(m_{\mathrm{v}}-M_{\mathrm{v}}\right)_{0}}=12.10 \pm 0.39$, a mean value of $\overline{E(B-V)}=0.30$, and an estimated age of 11.2 Myr.

Because distances to the clusters were derived by means of the $M_{\mathrm{V}}\left(\lambda_{1}, D\right)$ and $T_{\text {eff }}\left(\lambda_{1}, D\right)$-calibrations, this study also enables us to test the good behavior of the $M_{\text {bol }}\left(\lambda_{1}, D\right)$-calibration. On the other hand, it was also possible to detect systematic discrepancies between $\log g$ estimates from model atmospheres and those derived from stellar evolution models. We will discuss this parameter in more detail in a forecoming paper.

In summary, the BCD spectrophotometric system is a powerful tool for studying far galactic and extragalactic clusters with the generation of large telescopes and the multi-object technique, mainly because of the advantages of the BCD system, which is free from interstellar and circumstellar extinction. The method is also appropriate for studying and detecting stars with the Be phenomenon.

Acknowledgements. This research has made use of the SIMBAD database, operated at CDS, Strasbourg, France. We thank the referee, whose remarks helped to improve the present contribution greatly. We are grateful to R. Vazquez for his helpful comments and suggestions. L.C. acknowledges financial support from the Agencia Nacional de Promoción Científica y Tecnológica (BID 1728 OC/AR PICT 111), PIP 0300 CONICET, and the Programa de Incentivos G11/109 of the Universidad Nacional de La Plata, Argentina.

\section{References}

Ahmed, F. 1962, Publications of the Royal Observatory of Edinburgh, 3, 60 Allen, C. W. 1976, Astrophysical Quantities, 3rd edition (London: Athlone) Arp, H. C., \& van Sant, C. T. 1958, AJ, 63, 341

Balona, L., \& Crampton, D. 1974, MNRAS, 166, 203

Barbaro, G., Dallaporta, N., \& Nobili, L. 1967, Pubblicazioni dell'Osservatorio Astronomico di Padova, 138, 1

Becker, W. 1961, ZAp, 51, 151 
Becker, W. 1963, ZAp, 57, 117

Becker, W., \& Fenkart, R. 1971, A\&AS, 4, 241

Bertelli, G., Nasi, E., Girardi, L., \& Marigo, P. 2009, A\&A, 508, 355

Bidelman, W. P. 1954, PASP, 66, 249

Bressan, A., Fagotto, F., Bertelli, G., \& Chiosi, C. 1993, A\&AS, 100, 647

Buscombe, W. 1963, Mount Stromlo Observatory Mimeographs, 6, 0

Chalonge, D., \& Divan, L. 1973, A\&A, 23, 69

Chalonge, D., \& Divan, L. 1977, A\&A, 55, 117

Cidale, L., Zorec, J., \& Tringaniello, L. 2001, A\&A, 368, 160

Cidale, L. S., Arias, M. L., Torres, A. F., et al. 2007, A\&A, 468, 263

Crawford, D. L. 1978, AJ, 83, 48

Dachs, J., \& Kaiser, D. 1984, A\&AS, 58, 411

Deutschman, W. A., Davis, R. J., \& Schild, R. E. 1976, ApJS, 30, 97

Divan, L. 1954, Annales d'Astrophysique, 17, 456

Divan, L. 1979, in Spectral Classification of the Future, eds. M. F. McCarthy, A. G. D. Philip, \& G. V. Coyne, Ricerche Astronomiche, 9, IAU Colloq., 47, 247

Divan, L., \& Zorec, J. 1982, in The Scientific Aspects of the Hipparcos Space Astrometry Mission, ed. J. Delhaye, ESA Spec. Publ., 177, 101

Divan, L., Zorec, J., \& Andrillat, Y. 1983, A\&A, 126, L8

Dufton, P. L., Smartt, S. J., Lee, J. K., et al. 2006, A\&A, 457, 265

Eggen, O. J. 1977, PASP, 89, 187

Ekström, S., Georgy, C., Eggenberger, P., et al. 2012, A\&A, 537, A146

Evans, C. J., Smartt, S. J., Lee, J.-K., et al. 2005, A\&A, 437, 467

Feast, M. W. 1963, MNRAS, 126, 11

Flower, P. J. 1996, ApJ, 469, 355

Frémat, Y., Zorec, J., Hubert, A., \& Floquet, M. 2005, A\&A, 440, 305

Gerbaldi, M., \& Zorec, J. 1993, in Inside the Stars, eds. W. W. Weiss, \& A. Baglin (San Francisco: ASP), ASP Conf. Ser., 40, IAU Colloq., 137, 150

Głębocki, R., \& Gnaciński, P. 2005, in 13th Cambridge Workshop on Cool Stars, Stellar Systems and the Sun, eds. F. Favata, G. A. J. Hussain, \& B. Battrick, ESA SP, 560,571

Graham, J. A. 1967, MNRAS, 135, 377

Harris, G. L. H. 1976, ApJS, 30, 451

Hernández, C. 1959, Boletín de la Asociación Argentina de Astronomía, La Plata, Argentina, 2, 1

Hernández, C. 1960, Boletín de la Asociación Argentina de Astronomía, La Plata, Argentina, 2, 46
Huang, W., \& Gies, D. R. 2006, ApJ, 648, 580

Kennedy, P. M. 1966, Mount Stromlo Observatory Mimeographs, 9, 1

Kurucz, R. L. 1979, ApJS, 40, 1

Levesque, E. M., Massey, P., Olsen, K. A. G., et al. 2005, ApJ, 628, 973

McSwain, M. V., \& Gies, D. R. 2005, ApJS, 161, 118

McSwain, M. V., Huang, W., Gies, D. R., Grundstrom, E. D., \& Townsend, R. H. D. 2008, ApJ, 672, 590

Mermilliod, J. C. 1982, A\&A, 109, 48

Moitinho, A., Alfaro, E. J., Yun, J. L., \& Phelps, R. L. 1997, AJ, 113, 1359

Moujtahid, A., Zorec, J., Hubert, A. M., Garcia, A., \& Burki, G. 1998, A\&AS, 129,289

Neckel, T., Klare, G., \& Sarcander, M. 1980, A\&AS, 42, 251

Paunzen, E., \& Netopil, M. 2006, MNRAS, 371, 1641

Perry, C. L., Franklin, Jr., C. B., Landolt, A. U., \& Crawford, D. L. 1976, AJ, 81,632

Piatti, A. E., Claria, J. J., \& Bica, E. 1998, ApJS, 116, 263

Piatti, A. E., Clariá, J. J., \& Ahumada, A. V. 2011, New Astron., 16, 161

Rieke, G. H., \& Lebofsky, M. J. 1985, ApJ, 288, 618

Sanner, J., Brunzendorf, J., Will, J.-M., \& Geffert, M. 2001, A\&A, 369, 511

Savage, B. D., \& Mathis, J. S. 1979, ARA\&A, 17, 73

Schaller, G., Schaerer, D., Meynet, G., \& Maeder, A. 1992, A\&AS, 96, 269

Schild, R. E. 1970, ApJ, 161, 855

Schultz, G. V., \& Wiemer, W. 1975, A\&A, 43, 133

Sher, D. 1962, The Observatory, 82, 63

Sher, D. 1965, MNRAS, 129, 237

Shobbrook, R. R. 1984, MNRAS, 206, 273

Shobbrook, R. R. 1985, MNRAS, 212, 591

Shobbrook, R. R. 1987, MNRAS, 225, 999

Slettebak, A. 1985, ApJS, 59, 769

Tadross, A. L. 2001, New Ast., 6, 293

Wallenquist, A. 1959, Uppsala Astronomical Observatory Annals, 4

Whittet, D. C. B., \& van Breda, I. G. 1980, MNRAS, 192, 467

Winnenburg, W. 1973, A\&A, 24, 157

Yilmaz, F. 1976, A\&AS, 26, 1

Zorec, J. 1986, Thése d'État, Université de Paris, VII

Zorec, J., \& Briot, D. 1991, A\&A, 245, 150

Zorec, J., Frémat, Y., \& Cidale, L. 2005, A\&A, 441, 235

Zorec, J., Cidale, L., Arias, M. L., et al. 2009, A\&A, 501, 297 
Y. Aidelman et al.: Fundamental parameters of B stars

Table 7. NGC 3766: data obtained from interpolations in evolutionary diagrams given by Ekström et al. (2012) with rotation.

\begin{tabular}{|c|c|c|c|c|c|c|c|c|}
\hline $\begin{array}{l}\text { ID } \\
\text { Ahmed }\end{array}$ & $\begin{array}{c}\text { Other } \\
\text { designation }\end{array}$ & $\begin{array}{c}\log T_{\text {eff }} \\
{[\mathrm{K}]}\end{array}$ & $\log L / L_{\odot}$ & $M / M_{\odot}$ & $R / R_{\odot}$ & $\log g$ & $\begin{array}{c}\text { age } \\
{\left[10^{6} \mathrm{yr}\right]}\end{array}$ & $\log g_{\mathrm{BCD}}$ \\
\hline 1 & ALS 2398 & 4.209 & 4.03 & $8.7 \pm 0.2$ & $13.3 \pm 1.3$ & $3.13 \pm 0.09$ & $33.8 \pm 1.6$ & 2.81 \\
\hline 5 & HD 306794 & 4.358 & 4.58 & $12.0 \pm 1.8$ & $12.6 \pm 1.2$ & $3.31 \pm 0.15$ & $18.9 \pm 4.5$ & 3.06 \\
\hline 15 & ALS 2408 & 4.247 & 4.12 & $9.2 \pm 0.2$ & $12.3 \pm 1.2$ & $3.22 \pm 0.09$ & $29.9 \pm 1.2$ & 2.80 \\
\hline 24 & CPD-603131 & 4.179 & 2.37 & $2.1 \pm 1.4$ & $2.2 \pm 0.2$ & $4.06 \pm 0.37$ & $7.9 \pm 11.9$ & 4.24 \\
\hline 26 & CPD-603125 & 4.323 & 3.67 & $8.2 \pm 0.2$ & $5.2 \pm 0.5$ & $3.92 \pm 0.10$ & $25.7 \pm 5.0$ & 3.81 \\
\hline 27 & CPD-603128 & 4.313 & 3.67 & $8.1 \pm 0.2$ & $5.4 \pm 0.5$ & $3.88 \pm 0.10$ & $28.5 \pm 4.6$ & 3.49 \\
\hline 30 & CPD-603135 & 4.257 & 2.94 & $4.0 \pm 2.1$ & $3.0 \pm 0.3$ & $4.08 \pm 0.31$ & $16.2 \pm 14.3$ & 4.11 \\
\hline 55 & CPD-603138 & 4.100 & 2.46 & $3.7 \pm 0.1$ & $3.6 \pm 0.3$ & $3.90 \pm 0.09$ & $171.0 \pm 24.5$ & 4.02 \\
\hline 97 & CPD-603098 & 4.306 & 3.66 & $8.0 \pm 0.2$ & $5.5 \pm 0.5$ & $3.85 \pm 0.10$ & $30.4 \pm 4.6$ & 3.62 \\
\hline 151 & CD-603626 & 4.254 & 3.12 & $6.0 \pm 0.2$ & $3.8 \pm 0.4$ & $4.06 \pm 0.10$ & $39.2 \pm 12.1$ & 4.01 \\
\hline 169 & HD 100969 & 4.311 & 4.48 & $9.5 \pm 0.9$ & $13.8 \pm 1.3$ & $3.13 \pm 0.12$ & 18.5 & 2.80 \\
\hline 209 & HD 306800 & 4.314 & 3.30 & $7.0 \pm 0.2$ & $3.5 \pm 0.3$ & $4.19 \pm 0.10$ & $11.7 \pm 9.4$ & 4.03 \\
\hline 232 & HD 100943 & 4.195 & 5.19 & $19.8 \pm 0.8$ & $53.4 \pm 5.2$ & $2.28 \pm 0.10$ & $9.5 \pm 0.4$ & 2.80 \\
\hline 239 & HD 306798 & 4.307 & 3.64 & $7.9 \pm 0.2$ & $5.4 \pm 0.5$ & $3.88 \pm 0.10$ & $29.9 \pm 4.8$ & 3.62 \\
\hline 240 & ALS 2401 & 4.187 & 3.98 & $8.3 \pm 0.2$ & $13.8 \pm 1.3$ & $3.08 \pm 0.09$ & $36.6 \pm 1.7$ & 2.80 \\
\hline 243 & HD 308740 & 4.255 & 2.94 & $5.5 \pm 0.2$ & $3.1 \pm 0.3$ & $4.20 \pm 0.10$ & $19.5 \pm 15.5$ & 4.24 \\
\hline 264 & HD 306657 & 4.411 & 3.95 & $11.0 \pm 0.5$ & $4.8 \pm 0.5$ & $4.12 \pm 0.10$ & $7.8 \pm 4.2$ & 4.04 \\
\hline 291 & HD 306793 & 4.267 & 3.14 & $6.2 \pm 0.2$ & $3.6 \pm 0.3$ & $4.10 \pm 0.10$ & $31.1 \pm 12.3$ & 4.04 \\
\hline 301 & CPD-603087 & 4.259 & 3.14 & $6.1 \pm 0.2$ & $3.8 \pm 0.4$ & $4.07 \pm 0.10$ & $36.9 \pm 11.9$ & 4.01 \\
\hline 316 & BF Cen & 4.354 & 4.19 & $10.9 \pm 0.3$ & $8.2 \pm 0.8$ & $3.65 \pm 0.09$ & $19.6 \pm 1.9$ & 3.45 \\
\hline 317 & HD 306785 & 4.313 & 3.68 & $8.1 \pm 0.2$ & $5.5 \pm 0.5$ & $3.87 \pm 0.10$ & $28.5 \pm 4.5$ & 3.62 \\
\hline 326 & HD 100840 & 4.249 & 4.33 & $10.6 \pm 0.2$ & $15.6 \pm 1.5$ & $3.08 \pm 0.09$ & $22.90 \pm 3.7$ & 2.80 \\
\hline$\cdots$ & HD 306644 & 4.195 & 3.22 & $5.7 \pm 0.1$ & $5.6 \pm 0.5$ & $3.70 \pm 0.09$ & $73.4 \pm 7.3$ & 3.26 \\
\hline$\cdots$ & HD 306 & 4.308 & 3.47 & $7.4 \pm 0.2$ & $4.4 \pm 0.4$ & $4.02 \pm 0.10$ & $26.0 \pm 7.2$ & 4.00 \\
\hline$\cdots$ & HD 306784 & 4.119 & 2.28 & $3.6 \pm 0.1$ & $2.7 \pm 0.2$ & $4.14 \pm 0.10$ & $107.0 \pm 43.4$ & 4.03 \\
\hline$\cdots$ & HD 306787 & 4.210 & 2.84 & $5.0 \pm 0.1$ & $3.3 \pm 0.3$ & $4.09 \pm 0.10$ & $54.3 \pm 18.2$ & 4.11 \\
\hline$\cdots$ & HD 306788 & 4.303 & 3.44 & $7.3 \pm 0.2$ & $4.3 \pm 0.4$ & $4.02 \pm 0.10$ & $27.1 \pm 7.5$ & 4.01 \\
\hline$\ldots$ & HD 308743 & 4.250 & 3.10 & $5.9 \pm 0.2$ & $3.7 \pm 0.4$ & $4.06 \pm 0.10$ & $40.6 \pm 12.5$ & 4.01 \\
\hline$\cdots$ & HD 308852 & 4.192 & 3.00 & $5.2 \pm 0.1$ & $4.3 \pm 0.4$ & $3.87 \pm 0.09$ & $78.4 \pm 11.3$ & 3.83 \\
\hline$\cdots$ & NGC 3766 MG 29 & 4.260 & 3.15 & $6.1 \pm 0.2$ & $3.8 \pm 0.4$ & $4.07 \pm 0.10$ & $36.3 \pm 11.8$ & 4.11 \\
\hline ... & NGC 3766 MG 116 & 4.255 & 2.94 & $5.5 \pm 0.2$ & $3.1 \pm 0.2$ & $4.21 \pm 0.10$ & $18.8 \pm 15.4$ & 4.22 \\
\hline$\cdots$ & NGC 3766 MG 177 & 4.311 & 3.65 & $8.0 \pm 0.2$ & $5.3 \pm 0.5$ & $3.89 \pm 0.10$ & $29.0 \pm 4.8$ & 3.60 \\
\hline
\end{tabular}

Table 8. NGC 4755: data obtained from interpolations in evolutionary diagrams given by Ekström et al. (2012) with rotation.

\begin{tabular}{lcccccccc}
\hline \hline $\begin{array}{l}\text { ID } \\
\text { Arp }\end{array}$ & $\begin{array}{c}\text { Other } \\
\text { designation }\end{array}$ & $\begin{array}{c}\log T_{\text {eff }} \\
{[\mathrm{K}]}\end{array}$ & $\log L / L_{\odot}$ & $M / M_{\odot}$ & $R / R_{\odot}$ & $\log g$ & $\begin{array}{c}\text { age } \\
{\left[10^{6} \mathrm{yr}\right]}\end{array}$ & $\log g_{\mathrm{BCD}}$ \\
\hline 1 & NSV 6008 & 4.103 & 5.05 & $21.8 \pm 0.9$ & $69.9 \pm 6.8$ & $2.09 \pm 0.10$ & $8.8 \pm 0.3$ & 2.80 \\
5 & CPD-594552 & 4.316 & 5.45 & $24.6 \pm 0.6$ & $41.4 \pm 4.0$ & $2.59 \pm 0.09$ & $7.6 \pm 0.2$ & 2.80 \\
6 & ALS 2 816 & 4.415 & 4.40 & $13.2 \pm 0.4$ & $7.8 \pm 0.7$ & $3.77 \pm 0.10$ & $12.6 \pm 1.6$ & 3.25 \\
7 & BS Cru & 4.373 & 4.47 & $12.9 \pm 0.4$ & $10.3 \pm 1.0$ & $3.52 \pm 0.10$ & $15.4 \pm 1.3$ & 3.02 \\
8 & CPD-594540 & 4.306 & 3.24 & $5.0 \pm 2.6$ & $3.4 \pm 0.3$ & $4.07 \pm 0.31$ & $11.1 \pm 9.6$ & 4.22 \\
11 & CPD-594530 & 4.194 & 2.64 & $4.5 \pm 0.1$ & $2.9 \pm 0.3$ & $4.18 \pm 0.10$ & $47.2 \pm 24.4$ & 4.30 \\
106 & BU Cru & 4.314 & 5.44 & $24.5 \pm 0.6$ & $41.6 \pm 4.0$ & $2.59 \pm 0.09$ & $7.6 \pm 0.2$ & 2.80 \\
113 & CPD-594532 & 4.413 & 3.98 & $11.2 \pm 0.5$ & $4.9 \pm 0.5$ & $4.11 \pm 0.10$ & $7.9 \pm 4.0$ & 3.89 \\
117 & CPD-594531 & 4.306 & 3.23 & $4.8 \pm 2.7$ & $3.4 \pm 0.3$ & $4.06 \pm 0.33$ & $10.3 \pm 9.3$ & 4.21 \\
201 & EI Cru & 4.375 & 4.26 & $11.6 \pm 0.3$ & $8.0 \pm 0.8$ & $3.69 \pm 0.09$ & $16.8 \pm 1.8$ & 3.26 \\
202 & CX Cru & 4.412 & 4.14 & $11.8 \pm 0.4$ & $5.9 \pm 0.6$ & $3.97 \pm 0.10$ & $11.4 \pm 3.0$ & 3.81 \\
223 & CC Cru & 4.272 & 5.34 & $22.6 \pm 0.5$ & $44.8 \pm 4.3$ & $2.49 \pm 0.09$ & $8.3 \pm 0.2$ & 2.80 \\
305 & CN Cru & 4.494 & 4.36 & $9.6 \pm 7.0$ & $5.2 \pm 0.5$ & $3.99 \pm 0.40$ & $1.7 \pm 1.7$ & 4.12 \\
306 & CPD-594559 & 4.478 & 4.77 & $18.2 \pm 0.6$ & $9.1 \pm 0.9$ & $3.78 \pm 0.10$ & $7.7 \pm 1.0$ & 3.44 \\
418 & CPD-594542 & 4.363 & 4.39 & $12.2 \pm 0.4$ & $9.9 \pm 1.0$ & $3.53 \pm 0.10$ & $16.7 \pm 1.4$ & 3.02 \\
452 & HD 312 079 & 4.359 & 3.65 & $8.8 \pm 0.4$ & $4.3 \pm 0.4$ & $4.12 \pm 0.10$ & $12.6 \pm 6.4$ & 4.01 \\
454 & HD 312 080 & 4.362 & 3.78 & $9.2 \pm 0.3$ & $4.9 \pm 0.5$ & $4.02 \pm 0.10$ & $16.7 \pm 5.1$ & 3.83 \\
\hline
\end{tabular}


Table 12. NGC 3766: comparison among different $M_{\mathrm{v}}$ estimates.

\begin{tabular}{|c|c|c|c|c|c|}
\hline $\begin{array}{l}\text { ID } \\
\text { Ahmed }\end{array}$ & $\begin{array}{c}\text { Other } \\
\text { designation }\end{array}$ & This work & Sher & Balona & Shobbrook \\
\hline 1 & ALS 2398 (pe) & -4.05 & -1.26 & -2.00 & -3.75 \\
\hline 5 & HD 306794 & -4.56 & $\ldots$ & -3.70 & -3.88 \\
\hline 15 & ALS $2408(\mathrm{v})$ & -4.09 & $\ldots$ & -2.60 & -7.87 \\
\hline 24 & CPD-603131 & -0.02 & $\cdots$ & -1.30 & -1.01 \\
\hline 26 & CPD-603125 (ne) & -2.51 & $\ldots$ & -3.10 & $\ldots$ \\
\hline 27 & CPD-603128 & -2.58 & -1.10 & -3.10 & $\ldots$ \\
\hline 30 & CPD-603135 & -1.08 & -0.30 & -1.60 & -0.53 \\
\hline 55 & CPD-603138 & -0.54 & $\ldots$ & -1.00 & $\ldots$ \\
\hline 97 & CPD-603098 & -2.59 & $\ldots$ & -2.60 & $\ldots$ \\
\hline 151 & CD-603626 & -1.56 & $\ldots$ & -2.00 & $\ldots$ \\
\hline 169 & HD 100969 & -4.60 & -1.26 & -4.50 & -2.75 \\
\hline 209 & HD 306800 & -1.65 & $\ldots$ & -2.00 & -1.52 \\
\hline 232 & HD $100943^{*}(\mathrm{e})$ & $-7.00^{B}$ & $\ldots$ & -7.00 & -10.95 \\
\hline 239 & HD $306798(\mathrm{Be})$ & -2.53 & -1.10 & -2.60 & -2.37 \\
\hline 240 & ALS $2401^{*}(\mathrm{Be})$ & -4.01 & $\ldots$ & -3.60 & -3.84 \\
\hline 243 & HD 308740 & -1.11 & $\ldots$ & -1.30 & -0.66 \\
\hline 264 & HD $306657^{*}(\mathrm{Be})$ & -2.62 & $\ldots$ & -2.50 & $\ldots$ \\
\hline 291 & HD 306793 (e) & -1.53 & $\ldots$ & -2.00 & -3.94 \\
\hline 301 & CPD-603087 (e) & -1.57 & $\ldots$ & -2.00 & -3.08 \\
\hline 316 & BF Cen (A) & -3.60 & $\ldots$ & -3.70 & $\ldots$ \\
\hline 317 & HD 306785 & -2.59 & $\ldots$ & -3.70 & $\ldots$ \\
\hline 326 & HD 100840 & -4.61 & -1.26 & -3.90 & $\cdots$ \\
\hline$\cdots$ & HD 306644 & -2.10 & $\ldots$ & -1.70 & $\cdots$ \\
\hline .. & HD 3067 & -2.10 & $\ldots$ & -2.00 & $\ldots$ \\
\hline$\cdots$ & HD 306784 & -0.01 & $\ldots$ & -1.00 & $\ldots$ \\
\hline .. & HD 3067 & -1.06 & $\ldots$ & -1.30 & $\ldots$ \\
\hline$\cdots$ & HD 306788 & -2.06 & $\ldots$ & -2.00 & $\ldots$ \\
\hline$\cdots$ & HD 308743 & -1.52 & $\ldots$ & -2.00 & $\ldots$ \\
\hline$\ldots$ & HD $308852^{*}$ & -1.54 & $\ldots$ & -1.80 & $\ldots$ \\
\hline$\ldots$ & NGC 3766 MG 29 & -1.58 & $\ldots$ & -2.00 & $\ldots$ \\
\hline$\ldots$ & NGC 3766 MG 116 & -1.10 & $\ldots$ & -1.30 & $\ldots$ \\
\hline$\ldots$ & NGC 3766 MG 177 & -2.53 & $\ldots$ & -2.60 & $\ldots$ \\
\hline
\end{tabular}

Notes. (A) Algol eclipsing binary. (Be) Be star. (e) Emission line star. (v) Eruptive variable. (pe) Sharp $\mathrm{H} \gamma$ line and incipient emission at $\mathrm{H} \beta$. (ne) Moderate emission at $\mathrm{H} \beta .^{\left({ }^{*}\right)}$ Stars with double Balmer discontinuity. ${ }^{(B)}$ Balona \& Crampton (1974). 4th column: $M_{\mathrm{v}}$ values given by Sher (1965). 5th column: $M_{\mathrm{v}}$ values given by Balona \& Crampton (1974). 6th column: $M_{\mathrm{v}}$ values given by Shobbrook (1985).

Table 13. NGC 3766: comparison among individual estimates of $\left(m_{\mathrm{v}}-M_{\mathrm{v}}\right)_{0}$.

\begin{tabular}{lccccc}
\hline \hline $\begin{array}{l}\text { ID } \\
\text { Ahmed }\end{array}$ & $\begin{array}{c}\text { Other } \\
\text { designation }\end{array}$ & This work & Sher & Schild & Shobbrook \\
\hline 1 & ALS 2 398 (pe) & 11.97 & 9.27 & 11.10 & 11.71 \\
5 & HD 306 794 & 12.02 & $\ldots$ & 11.80 & 11.36 \\
15 & ALS 2 408 (v) & 11.72 & $\ldots$ & 11.20 & 15.68 \\
24 & CPD-60 3131 & 10.54 & $\ldots$ & $\ldots$ & 11.64 \\
26 & CPD-60 3125 (ne) & 11.57 & $\ldots$ & 11.90 & $\ldots$ \\
27 & CPD-60 3128 & 10.28 & 8.99 & 10.80 & $\ldots$ \\
30 & CPD-60 3135 & 11.97 & 10.83 & $\ldots$ & 11.01 \\
169 & HD 100 969 & 12.79 & 9.86 & $\ldots$ & 11.27 \\
209 & HD 306 800 & 11.31 & $\ldots$ & $\ldots$ & 11.48 \\
232 & HD 100 943*(e) & 13.50 & $\ldots$ & 13.30 & 17.40 \\
239 & HD 306 798 (Be) & 10.76 & 9.98 & $\ldots$ & 11.15 \\
240 & ALS 2 401*(Be) & 12.65 & $\ldots$ & $\ldots$ & 12.87 \\
243 & HD 308 740 & 10.91 & $\ldots$ & $\ldots$ & 10.80 \\
291 & HD 306 793 (e) & 11.14 & $\ldots$ & $\ldots$ & 13.85 \\
301 & CPD-60 3087 (e) & 12.47 & $\ldots$ & $\ldots$ & 13.20 \\
326 & HD 100 840 & 11.94 & 8.87 & $\ldots$ & $\ldots$ \\
\hline
\end{tabular}

Notes. (Be) Be star. (e) Emission line star. (v) Eruptive variable. (pe) Sharp $\mathrm{H} \gamma$ line and incipient emission at $\mathrm{H} \beta$. (ne) Moderate emission at $\mathrm{H} \beta$. ${ }^{(*)}$ Stars with double Balmer discontinuity. 4th column: $\left(m_{\mathrm{v}}-M_{\mathrm{v}}\right)_{0}$ values given by Sher (1965). 5th column: $\left(m_{\mathrm{v}}-M_{\mathrm{v}}\right)_{0}$ values given by Schild (1970). 6th column: $\left(m_{\mathrm{v}}-M_{\mathrm{v}}\right)_{0}$ values given by Shobbrook (1985). 
Table 14. NGC 3766: comparison among different $E(B-V)$ estimates.

\begin{tabular}{lccccc}
\hline \hline $\begin{array}{l}\text { ID } \\
\text { Ahmed }\end{array}$ & $\begin{array}{c}\text { Other } \\
\text { designation }\end{array}$ & This work & Sher & Schild & Shobbrook \\
\hline 1 & ALS 2 398 (pe) & 0.23 & 0.22 & 0.25 & 0.20 \\
5 & HD 306 794 & 0.23 & $\ldots$ & 0.07 & 0.22 \\
15 & ALS 2 408(v) & 0.29 & $\ldots$ & 0.31 & 0.23 \\
24 & CPD-603131 & 0.25 & $\ldots$ & $\ldots$ & 0.20 \\
26 & CPD-60 3125 (ne) & 0.00 & $\ldots$ & 0.17 & $\ldots$ \\
27 & CPD-603128 & 0.25 & 0.16 & 0.20 & $\ldots$ \\
30 & CPD-603135 & 0.07 & 0.18 & $\ldots$ & 0.19 \\
169 & HD 100 969 & 0.27 & 0.18 & $\ldots$ & 0.20 \\
209 & HD 306 800 & 0.37 & $\ldots$ & $\ldots$ & 0.22 \\
232 & HD 100 943*(e) & 0.21 & $\ldots$ & 0.26 & 0.23 \\
239 & HD 306 798 (Be) & 0.37 & 0.19 & $\ldots$ & 0.22 \\
240 & ALS 2 401*(Be) & 0.31 & $\ldots$ & $\ldots$ & 0.19 \\
243 & HD 308740 & 0.42 & $\ldots$ & $\ldots$ & 0.26 \\
264 & HD 306 657*(Be) & 0.43 & $\ldots$ & $\ldots$ & 0.24 \\
291 & HD 306 793 (e) & 0.34 & $\ldots$ & $\ldots$ & 0.24 \\
301 & CPD-60 3087 (e) & 0.00 & $\ldots$ & $\ldots$ & 0.23 \\
326 & HD 100 840 & 0.28 & 0.21 & $\ldots$ & $\ldots$ \\
\hline
\end{tabular}

Notes. (Be) Be star. (e) Emission line star. (v) Eruptive variable. (pe) Sharp $\mathrm{H} \gamma$ line and incipient emission at $\mathrm{H} \beta$. (ne) Moderate emission at $\mathrm{H} \beta$. ${ }^{(*)} \mathrm{S}$ tars with double Balmer discontinuity. 4th column: $\left(m_{\mathrm{v}}-M_{\mathrm{v}}\right)_{0}$ values given by Sher (1965). 5th column: $\left(m_{\mathrm{v}}-M_{\mathrm{v}}\right)_{0}$ values given by Schild (1970). 6th column: $\left(m_{\mathrm{v}}-M_{\mathrm{v}}\right)_{0}$ values given by Shobbrook (1985).

Table 16. NGC 4755: comparison among different $M_{\mathrm{v}}$ estimates.

\begin{tabular}{lcccccc}
\hline \hline $\begin{array}{l}\text { ID } \\
\text { Arp }\end{array}$ & $\begin{array}{c}\text { Other } \\
\text { designation }\end{array}$ & This work Balona & $\begin{array}{c}\text { Perry } \\
(\text { Cr })\end{array}$ & $\begin{array}{c}\text { Shobbrook } \\
(\text { Eggen })\end{array}$ & Shobbrook \\
\hline 1 & NSV 6 008* (vs) & $-7.00^{B}$ & -7.00 & -7.10 & -6.28 & -5.28 \\
5 & CPD-594552 & $-7.00^{B}$ & -7.00 & -4.40 & -4.26 & -3.76 \\
6 & ALS 2 816 (vc) & -3.70 & -4.10 & -3.90 & -3.54 & -3.14 \\
7 & BS Cru (vc) & -4.17 & -4.10 & -3.35 & -3.30 & -2.90 \\
8 & CPD-594540 & -1.54 & -2.00 & -3.75 & $\ldots$ & $\ldots$ \\
11 & CPD-594530 & -0.64 & -0.90 & -1.50 & -0.45 & -0.95 \\
106 & BU Cru & $-7.00^{B}$ & -7.00 & -6.15 & -5.81 & -5.01 \\
113 & CPD-594532 (vc) & -2.67 & -3.10 & -2.80 & -2.53 & -2.43 \\
117 & CPD-594531 & -1.52 & -2.00 & -2.35 & $\ldots$ & $\ldots$ \\
201 & EI Cru (vc) & -3.62 & -4.10 & -3.60 & -2.89 & -2.49 \\
202 & CX Cru (vc) & -3.07 & -3.10 & -2.95 & -3.23 & -3.03 \\
223 & CC Cru (ve) & $-7.00^{B}$ & -7.00 & -5.45 & -4.35 & -3.85 \\
305 & CN Cru (eb) & -3.07 & -3.80 & -4.60 & -3.04 & -2.64 \\
306 & CPD-594559* (Be) & -4.22 & -4.60 & -3.50 & $\ldots$ & $\ldots$ \\
418 & CPD-594542(vs) & -4.05 & -3.70 & -3.40 & -3.11 & -2.91 \\
452 & HD 312079 & -2.22 & -2.50 & $\ldots$ & $\ldots$ & $\ldots$ \\
454 & HD 312080 & -2.52 & -3.10 & $\ldots$ & $\ldots$ & $\ldots$ \\
\hline
\end{tabular}

Notes. (Be) Be star. (vs) Variable star. (vc) Variable star of beta Cep type. (ve) Ellipsoidal variable star. (eb) Eclipsing binary of beta Lyr type. ${ }^{(*)}$ Stars with double Balmer discontinuity. ${ }^{(B)} M_{\mathrm{v}}$ values adopted from Balona \& Crampton (1974). 4th column: $M_{\mathrm{v}}$ derived from Balona \& Crampton (1974). 5th column: $M_{\mathrm{v}}$ values given by Perry et al. (1976). 6th and 7th column: $M_{\mathrm{v}}$ ) values given by Shobbrook (1984). 
A\&A 544, A64 (2012)

Table 17. NGC 4755: comparison among individual estimates of $\left(m_{\mathrm{v}}-M_{\mathrm{v}}\right)_{0}$.

\begin{tabular}{lcccc}
\hline \hline $\begin{array}{l}\text { ID } \\
\text { Arp }\end{array}$ & $\begin{array}{c}\text { Other } \\
\text { designation }\end{array}$ & This work & $S_{\text {Cr }}$ & $S_{\text {Eggen }}$ \\
\hline 1 & NSV 6008*(vs) & 11.59 & 10.70 & 9.70 \\
5 & CPD-594552 & 14.79 & 11.30 & 10.80 \\
6 & ALS 2 816 (vc) & 11.51 & 11.50 & 11.10 \\
7 & BS Cru (vc) & 13.39 & 11.80 & 11.40 \\
8 & CPD-594540 & 10.31 & $\ldots$ & $\ldots$ \\
11 & CPD-594530 & 10.66 & 10.80 & 11.30 \\
106 & BU Cru & 13.02 & 11.40 & 10.60 \\
113 & CPD-594532(vc) & 12.14 & 11.50 & 11.40 \\
117 & CPD-594531 & 11.20 & $\ldots$ & $\ldots$ \\
201 & EI Cru (vc) & 12.45 & 11.20 & 10.80 \\
202 & CX Cru (vc) & 12.40 & 12.20 & 12.00 \\
223 & CC Cru (ve) & 13.91 & 10.80 & 10.30 \\
305 & CN Cru (eb) & 10.32 & 10.30 & 9.90 \\
306 & CPD-594559* (Be) & 12.29 & $\ldots$ & $\ldots$ \\
418 & CPD-594542 (vs) & 13.19 & 11.60 & 11.40 \\
452 & HD 312079 & 11.49 & $\ldots$ & $\ldots$ \\
454 & HD 312080 & 11.86 & $\ldots$ & $\ldots$ \\
\hline
\end{tabular}

Notes. (Be) Be star. (vs) Variable star. (vc) Variable star of beta Cep type. (ve) Ellipsoidal variable star. (eb) Eclipsing binary of beta Lyr type. ${ }^{(*)}$ Stars with double Balmer discontinuity. $S_{\mathrm{Cr}}$ : Shobbrook (1984), using calibration $\beta / M_{\mathrm{v}}$ given by Crawford (1978). $S_{\text {Eggen }}$ : Shobbrook (1984), using calibration $\beta /[u-b] / M_{\mathrm{v}}$ given by Eggen (1977).

Table 18. NGC 4755: comparison among different $E(B-V)$ estimates.

\begin{tabular}{lcccc}
\hline \hline $\begin{array}{l}\text { ID } \\
\text { Arp }\end{array}$ & $\begin{array}{c}\text { Other } \\
\text { designation }\end{array}$ & This work & Perry & Shobbrook \\
\hline 1 & NSV 6008*(vs) & 0.38 & 0.34 & 0.42 \\
5 & CPD-594552 & 0.16 & 0.43 & 0.41 \\
6 & ALS 2 816 (vc) & 0.39 & 0.36 & 0.35 \\
7 & BS Cru (vc) & 0.17 & 0.42 & 0.39 \\
8 & CPD-594540 & 0.36 & 0.50 & 0.43 \\
11 & CPD-594530 & 0.44 & 0.36 & 0.34 \\
106 & BU Cru & 0.29 & 0.40 & 0.41 \\
113 & CPD-594532 (vc) & 0.22 & 0.38 & 0.38 \\
117 & CPD-594531 & 0.39 & 0.45 & 0.43 \\
201 & EI Cru (vc) & 0.18 & 0.39 & 0.35 \\
202 & CX Cru (vc) & 0.22 & 0.39 & 0.34 \\
223 & CC Cru (ve) & 0.30 & 0.50 & 0.47 \\
305 & CN Cru (eb) & 0.43 & 0.41 & 0.38 \\
306 & CPD-594559* (Be) & 0.62 & 0.52 & 0.50 \\
418 & CPD-594542 (vs) & 0.17 & 0.41 & 0.38 \\
452 & HD 312 079 & 0.25 & $\ldots$ & $\ldots$ \\
454 & HD 312 080 & 0.20 & $\ldots$ & $\ldots$ \\
\hline
\end{tabular}

Notes. (Be) Be star. (vs) Variable star. (vc) Variable star of beta Cep type. (ve) Ellipsoidal variable star. (eb) Eclipsing binary of beta Lyr type. (*) Stars with double Balmer discontinuity. 4th column: $E(B-V)$ values given by Perry et al. (1976). 5th column: $E(B-V)$ values given by Shobbrook (1984). 